\title{
IUCrJ
}

Volume 7 (2020)

Supporting information for article:

The structure of kaliophilite KAISiO4, a long-lasting crystallographic problem

Enrico Mugnaioli, Elena Bonaccorsi, Arianna E. Lanza, Erik Elkaim, Virginia DiezGómez, Isabel Sobrados, Mauro Gemmi and Miguel Gregorkiewitz 


\section{The structure of kaliophilite $\mathrm{KAISiO}_{4}$, a long-lasting crystallographic problem}

Enrico Mugnaioli ${ }^{\mathrm{a}}$, Elena Bonaccorsi ${ }^{\mathrm{b} *}$, Arianna E. Lanza ${ }^{\mathrm{a}}$, Erik Elkaim ${ }^{\mathrm{c}}$, Virginia Diez-Gómez $^{\mathrm{d}}$, Isabel Sobrados ${ }^{\mathrm{d}}$, Mauro Gemmi* ${ }^{\mathrm{a} *}$, Miguel Gregorkiewitz ${ }^{\mathrm{e}}$

${ }^{a}$ Center for Nanotechnology Innovation@NEST, Istituto Italiano di Tecnologia, Piazza S. Silvestro 12, Pisa, 56127 , Italy

bDipartimento di Scienze della Terra, Università di Pisa, Via Santa Maria 53, Pisa, 56126, Italy

'Synchrotron Soleil, L'Orme des Merisiers, Saint-Aubin, Gif-sur-Yvette, 91192, France

dinstituto de Ciencia de Materiales de Madrid, Consejo Superior de Investigaciones Científicas (ICMMCSIC), Sor Juana Inés de la Cruz 3, Madrid, 28049, Spain

'Dipartimento di Scienze Fisiche, della Terra e dell'Ambiente, Università di Siena, Via Laterina 8, Siena, 53100, Italy

Correspondence email: elena.bonaccorsi@unipi.it; mauro.gemmi@iit.it; gregormigu@gmail.com

\section{Supporting Information:}

S1. Ring types in $6^{3}$ nets; chemical composition

S2. ${ }^{29} \mathrm{Si}$ and ${ }^{27} \mathrm{Al}$ MAS-NMR spectra

S3. Optical microscopy and Weissenberg X-ray diffraction patterns

S4. High resolution synchrotron single crystal diffraction scans

S5. Crystal data, atom parameters, T-O and K-O interatomic distances

S6. Rietveld refinements using high resolution synchrotron radiation

S7. Pseudo-extinction rule 
S1. Ring types in $6^{3}$ nets; chemical composition

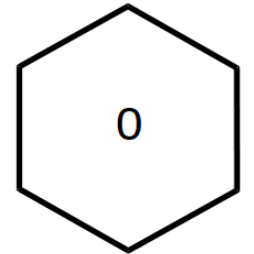

DDDDDD

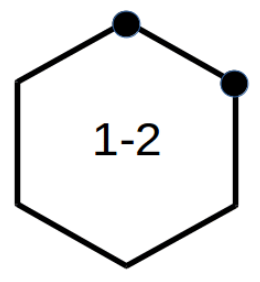

UUDDDD

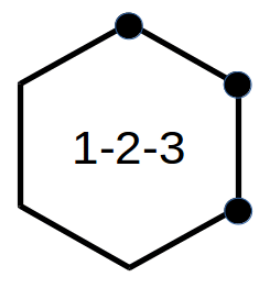

UUUDDD

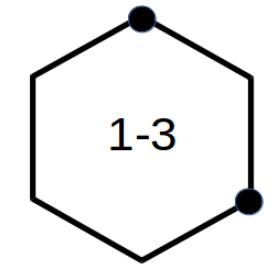

UDUDDD

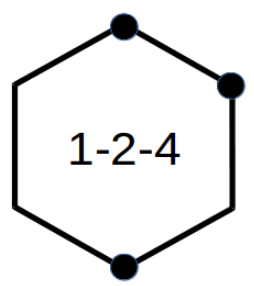

UUDUDD

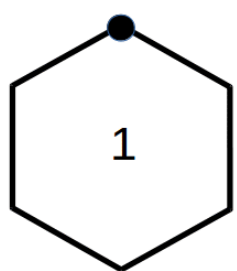

UDDDDD

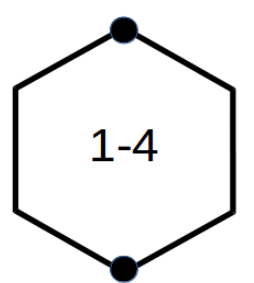

UDDUDD

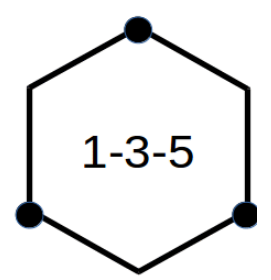

UDUDUD

Figure S1.1. Possible types of six-membered rings of tetrahedral nodes. In a $6^{3}$ net, all nodes are linked to three neighbours in the basal plane. Dots stand for nodes where the apical vertex points upwards (U), the remainder points downwards (D). 
Table S1.1. Published chemical analyses, densities and optical constants of kaliophilite (wet analyses except the last five). All values from original paper except sca88 and zam 10 for which 2 secondary references (mug27 and ban31) have been used. All elements have been recalculated from oxides per 4 oxygens.

\begin{tabular}{|c|c|c|c|c|c|c|c|c|c|c|c|c|c|c|c|c|}
\hline $\begin{array}{l}\text { Locality } \\
\text { Oxides (ms }\end{array}$ & $\begin{array}{l}\text { Vesuv } \\
\text { ass\%) }\end{array}$ & Somma & Vesuv & Vesuv & Somma & Vesuv & Vesuv & Somma & $\mathrm{CC}$ & $\mathrm{CC}$ & Ariccia & Somma & $\mathrm{CC}$ & Vesuv & $\mathrm{MF}$ & $\mathrm{MF}$ \\
\hline $\mathrm{SiO} 2$ & 40.43 & 37.45 & 37.73 & 38.53 & 39.04 & 38.0 & 39.2 & 38.62 & 38.17 & 38.73 & 39.05 & 38.87 & 39.41 & 38.73 & 38.0 & 38.2 \\
\hline $\mathrm{A} 12 \mathrm{O} 3$ & 33.07 & 32.43 & 33.09 & 32.20 & 31.96 & 28.8 & 33.36 & 32.09 & 31.73 & 31.04 & 30.31 & 31.89 & 32.75 & 31.55 & 30.2 & 31.3 \\
\hline $\mathrm{Fe} 2 \mathrm{O} 3$ & - & - & - & 0.12 & 0.98 & - & - & 0.80 & 0.18 & $\operatorname{tr}$ & $\operatorname{tr}$ & - & - & - & 2.22 & 0.77 \\
\hline $\mathrm{FeO}$ & - & - & - & - & - & - & - & 0.29 & 0 & 0 & 0 & 0.07 & 0.34 & 0.21 & 0 & 0 \\
\hline $\mathrm{MgO}$ & - & - & - & $\operatorname{tr}$ & 0.15 & - & - & 0 & $\operatorname{tr}$ & 0 & 0 & - & 0 & 0 & 0 & 0 \\
\hline $\mathrm{CaO}$ & 3.07 & 2.18 & - & 0.28 & 0.33 & 0.5 & 0.47 & 0 & 1.87 & 2.39 & 1.09 & 0.05 & 0 & 0 & 0 & 0.11 \\
\hline $\mathrm{Na} 2 \mathrm{O}$ & - & 2.26 & 0.37 & 2.12 & 3.89 & $\operatorname{tr}$ & 2.88 & 3.77 & 2.17 & 0.77 & 1.68 & 0.77 & 3.26 & 1.04 & 1.28 & 1.35 \\
\hline $\mathrm{K} 2 \mathrm{O}$ & 22.25 & 27.20 & 29.30 & 26.62 & 22.84 & 32.2 & 24.13 & 24.18 & 24.56 & 23.97 & 25.75 & 28.76 & 24.07 & 28.07 & 27.3 & 27.7 \\
\hline $\mathrm{H} 2 \mathrm{O}$ & 1.18 & - & & - & 0.60 & - & - & 0.23 & 1.23 & 2.98 & 1.88 & - & - & - & - & - \\
\hline total & 100.00 & 101.52 & 100.49 & $100.16^{1}$ & 99.79 & 99.5 & 100.04 & 99.98 & $99.95^{2}$ & $99.96^{3}$ & 99.80 & 100.41 & 99.83 & 99.6 & 99.00 & $99.62^{4}$ \\
\hline \multicolumn{17}{|c|}{ Elements per 4 oxygens } \\
\hline $\mathrm{Si}$ & 1.03 & 0.97 & 0.99 & 1.00 & 1.01 & 1.03 & 1.00 & 1.00 & 1.00 & 1.03 & 1.04 & 1.015 & 1.01 & 1.02 & 1.01 & 1.01 \\
\hline $\mathrm{Al}$ & 0.99 & 0.99 & 1.02 & 0.99 & 0.98 & 0.92 & 1.01 & 0.98 & 0.98 & 0.97 & 0.95 & 0.981 & 0.99 & 0.98 & 0.94 & 0.97 \\
\hline $\mathrm{Fe} 3+$ & - & - & - & $\operatorname{tr}$ & 0.02 & - & - & 0.02 & $\operatorname{tr}$ & $\operatorname{tr}$ & $\operatorname{tr}$ & - & - & & 0.04 & 0.02 \\
\hline $\mathrm{Fe} 2+$ & - & - & - & - & - & - & - & 0.01 & 0 & 0 & 0 & 0.001 & 0.01 & 0.01 & 0 & 0 \\
\hline $\mathrm{Ca}$ & 0.08 & 0.06 & - & 0.01 & 0.01 & 0.01 & 0.01 & 0 & 0.05 & 0.07 & 0.03 & 0.002 & 0 & 0 & 0 & 0 \\
\hline $\mathrm{Na}$ & - & 0.11 & 0.02 & 0.11 & 0.20 & $\operatorname{tr}$ & 0.14 & 0.19 & 0.11 & 0,04 & 0,09 & 0.039 & 0.16 & 0.05 & 0.07 & 0.07 \\
\hline $\mathrm{K}$ & 0.72 & 0.90 & 0.98 & 0.89 & 0.75 & 1.11 & 0.79 & 0.80 & 0.82 & 0.81 & 0.87 & 0.956 & 0.79 & 0.94 & 0.92 & 0.93 \\
\hline \multicolumn{17}{|c|}{ Density $\left(\mathrm{g} / \mathrm{cm}^{3}\right)$} \\
\hline$\rho$ & 2.67 & 2.602 & 2.493 & 2.628 & 2.61 & 2.598 & 2.606 & & & & & & & & & \\
\hline \multicolumn{17}{|c|}{ Optical properties } \\
\hline$\varepsilon$ & & & & & 1.5273 & 1.527 & 1.5258 & 1.5303 & 1.5312 & 1.5289 & 1.5294 & & & & & \\
\hline$\omega$ & & & & & 1.5316 & 1.531 & 1.5296 & 1.5345 & 1.5362 & 1.5338 & 1.5349 & & & & & \\
\hline$\Delta \mathrm{n}=\varepsilon-\omega$ & & $<0$ & & & -0.0043 & -0.004 & -0.0038 & -0.0042 & -0.0050 & -0.0049 & -0.0055 & & & & & \\
\hline Reference & $\operatorname{cov} 39$ & mie87 & sca88 & zam10 & $\operatorname{mug} 27$ & ban31 & ban31 & $\operatorname{sah} 62^{5}$ & bar70 & bar70 & bar70 & abb84 & aur85 & cel92 & dib01 & dib01 \\
\hline
\end{tabular}

Abbreviated localities: Vesuv = Vesuvius, $\mathrm{CC}=$ Colle Cimino, $\mathrm{MF}=$ Montefiascone. - stays for not determined; 0 stays for below detection limit / not detected; tr stays for traces; ${ }^{1}$ : contains $0.14 \% \mathrm{Cl},{ }^{2}$ : contains $0.04 \% \mathrm{Rb}_{2} \mathrm{O},{ }^{3}$ : contains $0.08 \% \mathrm{Rb}_{2} \mathrm{O} ;{ }^{4}$ : contains traces of $\mathrm{Mn} ;{ }^{5}$ : optical properties determined by bar70.

References. abb84: Abbott (1984); aur85: Aurisicchio \& Federico (1985); ban31: Bannister \& Hey (1931); bar70: Barbieri et al. (1970); cel92: Cellai et al. (1992); cov39: Covelli (1839); dib01: Di Battistini et al. (2001); mie87: Mierisch (1887); mug27: Mügge (1927); sah62: Sahama (1962); sca88: Scacchi (1888); zam10: Zambonini (1910). 


\section{References}

Abbott Jr., R. N. (1984). Am. Mineral. 69, 449-457.

Aurisicchio, C. \& Federico, M. (1985). Bull. Geol. Soc. Finland 57, 129-137.

Bannister, F. A. \& Hey, M. H. (1931). Mineral. Mag. 22, 569-608.

Barbieri, M., Federico, M. \& Tolomeo, L. (1970). Period. Miner. 39, 323-341 (in Italian)

Cellai, D., Carpenter, M. A. \& Heaney, P. J. (1992). Eur. J. Mineral. 4, 1209-1220.

Covelli, N. (1839). Atti Acc. Sc. Napoli 4, 17-32 (in Italian).

Di Battistini, G., Montanini, A., Vernia, L., Venturelli, G. \& Tonarini, S. (2001). Lithos 59, 1-24.

Mierisch, B. (1887). Tschermak's Miner. Petr. Mittheil. 8, 113-189 (in German).

Mügge, O. (1927). Z. Kristallogr. 65, 381-390 (in German).

Sahama, T. G. (1962). Norsk Geol. Tidsskrift 42, 168-179.

Scacchi, E. (1888). Rend. Accad. Fis. Mat. Napoli ser. 2, 2, 487 (in Italian).

Zambonini, F. (1910). Rend. Accad. Fis. Mat. Napoli 49, 83 (in Italian). 


\section{S2. ${ }^{29} \mathrm{Si}$ and ${ }^{27} \mathrm{Al}$ MAS-NMR spectra}
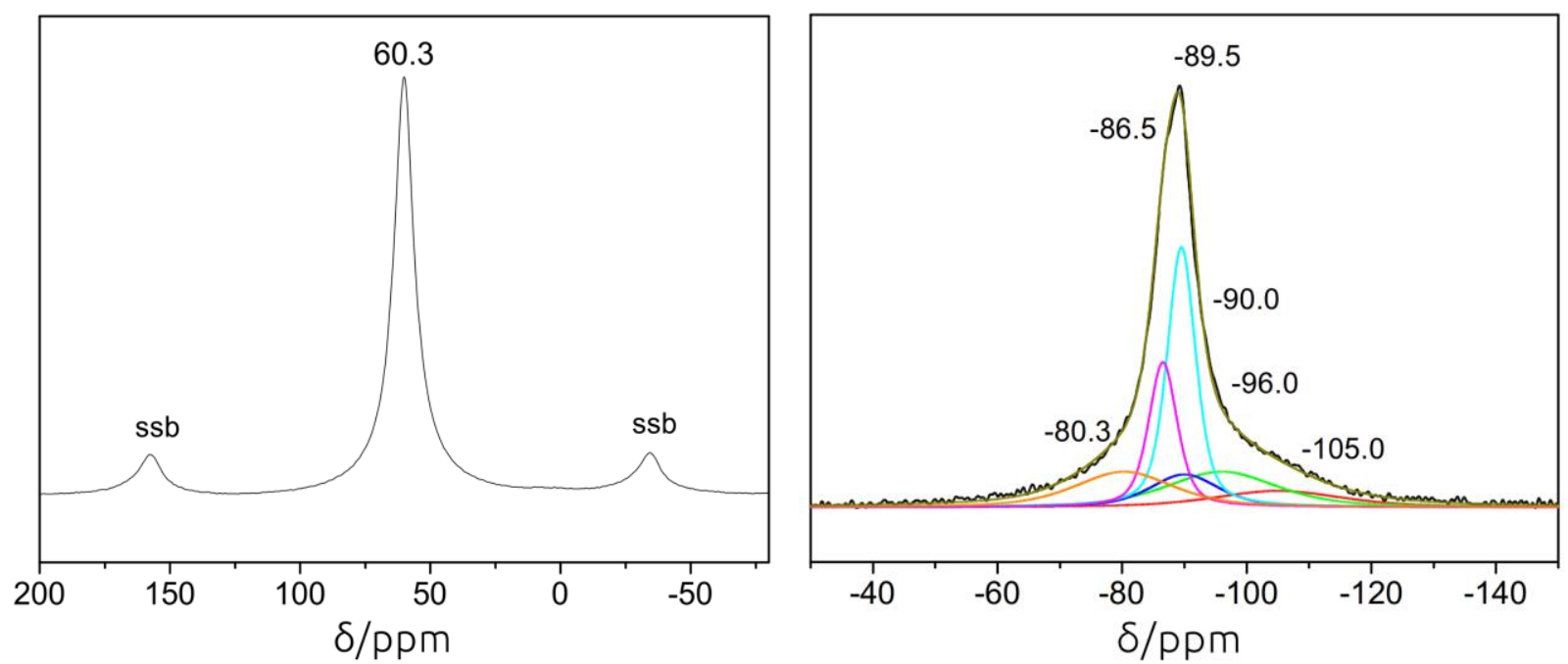

Figure S2.1. ${ }^{27} \mathrm{Al}$ (left) and ${ }^{29} \mathrm{Si}$ MAS-NMR (right) spectra of kaliophilite from Colle Cimino (sample cc1); black observed, yellow sum of contributions. ssb indicates spinning sidebands.
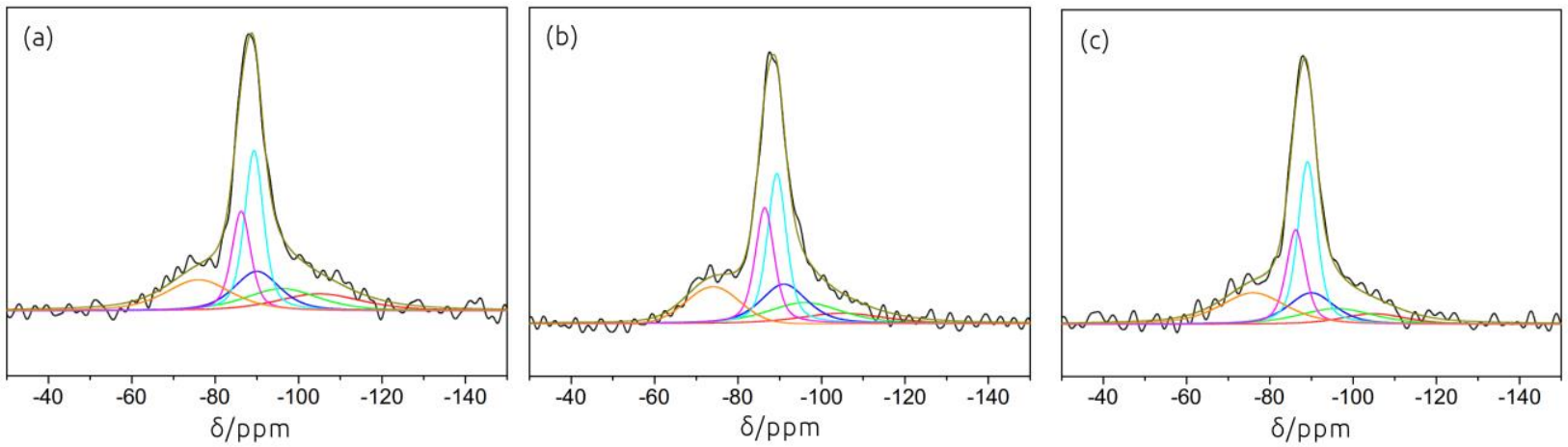

Figure S2.2. ${ }^{29} \mathrm{Si}$ MAS-NMR spectra of kaliophilite from three different samples from Colle Cimino: \#C1 (a), \#CL (b) and \#IH (c).

${ }^{29} \mathrm{Si}$ MAS-NMR spectra were obtained for various kaliophilite samples from Colle Cimino, using exceptionally long accumulation times to allow interpretation of the broad base of the peaks. The spectra of the four samples in Fig. S2.1 and Fig. S2.2 show clearly that there is a difference in the left, but not in the right part of the base. We therefore concluded that the left part is probably due to some impurity which changes from sample to sample, while the right part is inherent to kaliophilite itself.

To confirm the hypothesis, we simulated a spectrum containing six components: a central doublet due to kaliophilite $(\delta=-86.5$ and $89.5 \mathrm{ppm})$, and four broad peaks representing an impurity at about -74 to $-80 \mathrm{ppm}$, a sum of distorted kaliophilite $\mathrm{Q}^{4}(4 \mathrm{Al})$ environments at around $-90 \mathrm{ppm}$, and two environments resulting from $\mathrm{Si}$ for $\mathrm{Al}$ substitution in kaliophilite, i.e. $\mathrm{Q}^{4}(3 \mathrm{Al})$ at about $-96 \mathrm{ppm}$ and $\mathrm{Q}^{4}(0 \mathrm{Al})$ at about $-105 \mathrm{ppm}$. The latter two have been found earlier for Bancroft nepheline and its K-exchanged derivative (Stebbins et al., 1986; Sobrados, 1991) and are thought to be associated with alkali vacancies and twin boundaries. All peaks were found from curve fitting with some restriction for the -96 and $-105 \mathrm{ppm}$ positions. Fitting results are summarized in Table S2.1.

The central doublet was also observed in the ${ }^{29} \mathrm{Si}$ MAS-NMR spectrum of $O 1$ and the amount of disordered $\mathrm{Q}^{4}(4 \mathrm{Al})$ and $\mathrm{Q}^{4}(3 \mathrm{Al})$ and $\mathrm{Q}^{4}(0 \mathrm{Al})$ environments in kaliophilite is compatible with earlier findings for nepheline and $O 1$ (Stebbins et al., 1986; Gregorkiewitz et al., 2008). The amount of impurities, on the other hand, seems 
high and this is not supported by powder diffraction where crystalline impurities can be excluded at a level of about $0.4 \%$, and amorphous impurities seem improbable.

Table S2.1. Chemical shifts $\delta$, widths and areas as obtained from fitting of the six ${ }^{29} \mathrm{Si}$ peaks in Fig. S2.1 and Fig. S2.2. Samples are cc1 ( $1^{\text {st }}$ entry), \#C1 ( $2^{\text {nd }}$ entry), \#CL ( $3^{\text {rd }}$ entry) and \#IH (4th entry). Kp stands for kaliophilite.

\begin{tabular}{|l|l|l|l|l|l|l|}
\hline & "impurity" & \multicolumn{2}{|c|}{$\mathrm{Kp} \mathrm{Q}^{4}(4 \mathrm{Al})$} & $\mathrm{Kp} \mathrm{Q} \mathrm{Q}^{4}(4 \mathrm{Al})$ dis & $\mathrm{Kp} \mathrm{Q}^{4}(3 \mathrm{Al})$ & $\mathrm{Kp} \mathrm{Q}(0 \mathrm{Al})$ \\
\hline$\delta / \mathrm{ppm}$ & -80 & -86.5 & -89.5 & -90 & -96 & -105 \\
& -76 & -86.2 & -89.3 & -90 & -96 & -105 \\
& -74 & -86.4 & -89.3 & -91 & -96 & -105 \\
& -76 & -86.2 & -89.1 & -90 & -96 & -105 \\
\hline FWHM/ppm & 19 & 5.4 & 5.4 & 13 & 21 & 24 \\
& 19 & 5.4 & 5.4 & 13 & 21 & 24 \\
& 15 & 5.4 & 5.4 & 13 & 21 & 24 \\
& 19 & 5.4 & 5.4 & 13 & 21 & 18 \\
\hline area/\% & 14.7 & 18.1 & 32.5 & 9.7 & 16.4 & 8.6 \\
& 16.9 & 16.4 & 26.3 & 15.2 & 13.4 & 11.8 \\
& 14.8 & 20.6 & 26.6 & 16.5 & 13.8 & 7.7 \\
& 19.8 & 17.9 & 30.9 & 14.1 & 11.0 & 6.4 \\
\hline
\end{tabular}

The central kaliophilite doublet (Fig. S2.3) was calculated ab initio using CASTEP (Clark et al., 2005) for the model given in Table S5.3, which contains 18 crystallographically different Si positions, space group P3. While the positions are very similar to the fitting results, intensities appear inverted with the higher peak at about $-86.4 \mathrm{ppm}$. The nuclei probed in NMR are different from those in XRD, and small differences in the structures of the Colle Cimino and Pollena samples are possible.

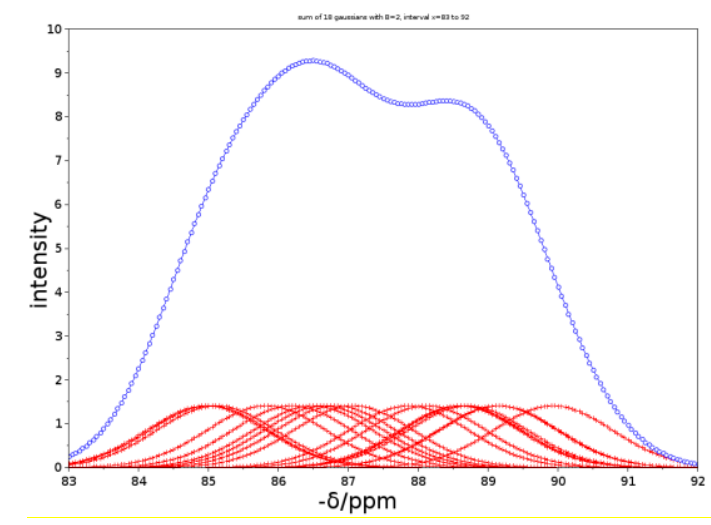

Figure S2.3. The central doublet in the ${ }^{29} \mathrm{Si}$ MAS-NMR spectrum of kaliophilite as calculated $a b$ initio from CASTEP. Gaussian curves with $F W H M=2$ for the 18 single contributions have been used. 


\section{References}

Clark, S. J., Segall, M. D., Pickard, C. J., Hasnip, P. J., Probert, M. J., Refson, K. \& Payne, M. C. (2005). Z. Kristallogr. 220, 567-570.

Gregorkiewitz, M., Li, Y., White, T. J., Withers, R. L. \& Sobrados I. (2008). Can. Mineral. 46, 1511-1526. Sobrados, I. (1991). PhD Thesis, Universidad Complutense, Madrid (in Spanish).

Stebbins, J. F., Murdoch, J. B., Carmichael, I. S. E. \& Pines, A. (1986). Phys. Chem. Mineral. 13, 371-381. 


\section{S3. Optical microscopy and Weissenberg X-ray diffraction patterns}

On the search of possibly pure single crystals, about 12 optically homogeneous microcrystals could be isolated under the petrographic microscope (Fig. S3.1). With an approximate thickness of $40 \mu \mathrm{m}$, the observed interference colours indicate $\Delta n \simeq-0.004$, in agreement with literature (Table S1.1). Although clear, many crystals show lines along the needle which are probably twin planes (see also similar observations in Cellai et al., 1992), but artefacts cannot be excluded for such small dimensions.

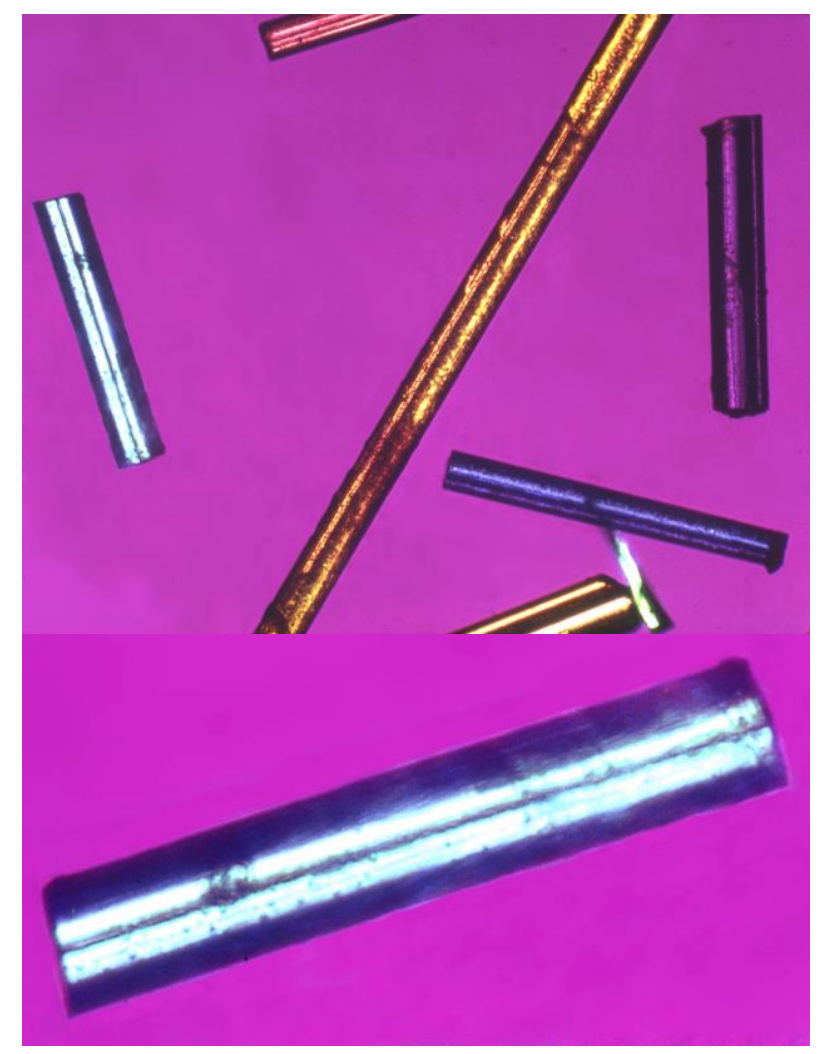

Figure S3.1. Microscopic view of kaliophilite needles from Colle Cimino (crossed polarizers, red I auxiliary plate). The diameter of the magnified needle is about $40 \mu \mathrm{m}$. Prismatic faces are clearly visible. In the termination of the hexagonal prisms, basal faces appear to prevail over pyramidal faces.

Further screening of these crystals with laboratory single crystal diffraction revealed that many of them were composed of slightly $\left(\sim 1^{\circ}\right)$ disoriented individuals but no streaks parallel or orthogonal to c could be detected. A representative Weissenberg pattern is given in Fig. S3.2.

For one crystal, $\mathrm{CuK \alpha}$ Weissenberg photographs of levels $h k l: 0 \leq l \leq 5$ showed no splitting. In order to test symmetry and twinning, this crystal was used to collect high resolution diffraction scans (Supporting Information S4). 

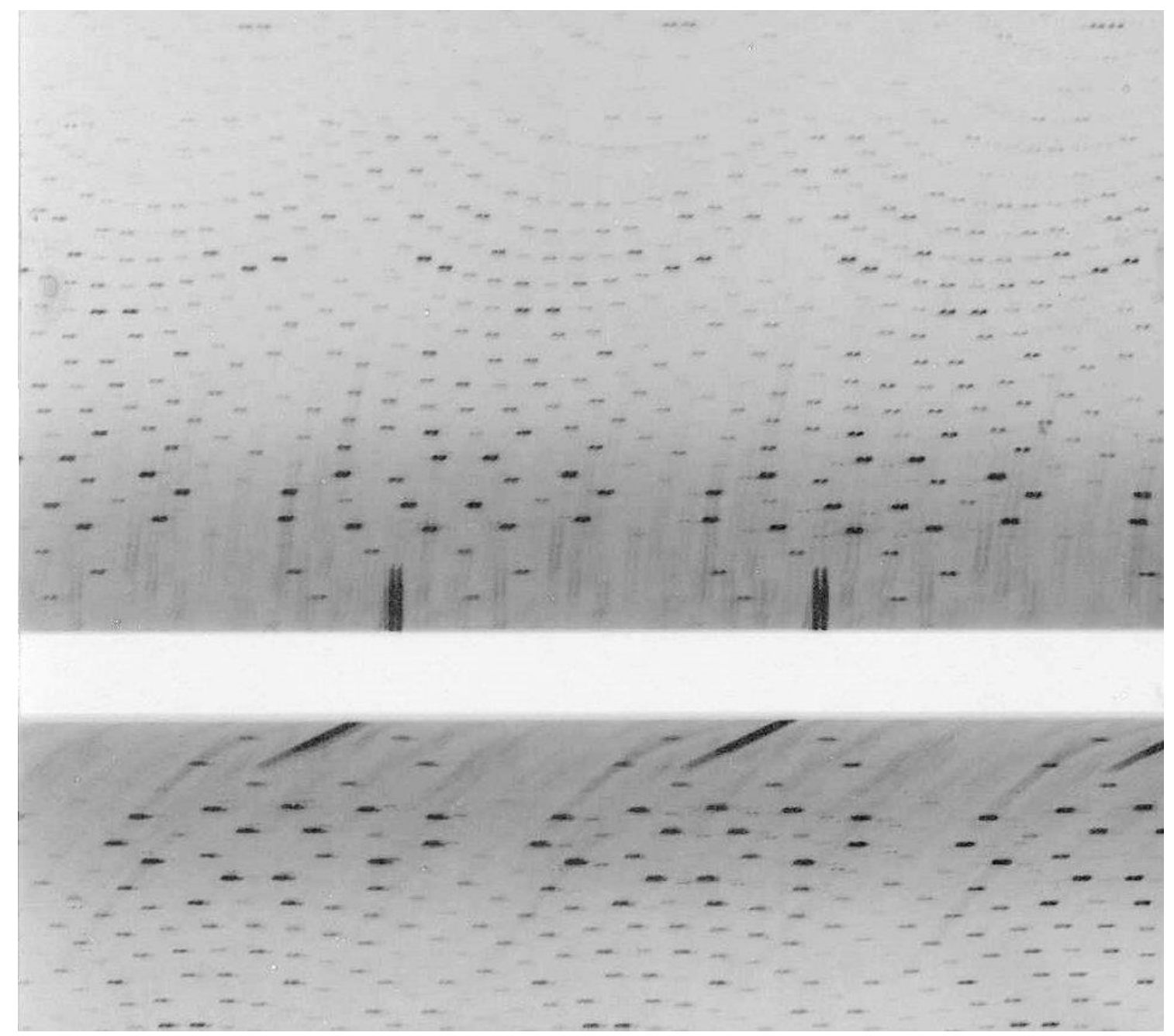

Figure S3.2. Level $h k 1$ Weissenberg equi-inclination diagram obtained with Ni-filtered $\mathrm{CuK}$ radiation $(\lambda=$ $1.5406 \AA$, camera $R=27.65 \mathrm{~mm}, 2^{\circ}(\omega) / \mathrm{mm}$ translation $\left.\| \mathbf{c}\right)$ for a kaliophilite 'single' crystal. Abscissa gives crystal rotation, ordinate gives diffraction angle. The $\mathbf{a}^{*}$ repeat lies on the almost extinct line passing through the abscissa. Splitting of reflections shows that the 'crystal' is composed of essentially two individuals which are rotated by $\Delta \omega<1.4^{\circ}$ against each other. Note that streaks are due to white radiation and not to structural features. 


\section{References}

Cellai, D., Carpenter, M. A. \& Heaney, P. J. (1992). Eur. J. Mineral. 4, 1209-1220. 


\section{S4. High resolution synchrotron single crystal diffraction scans}

1D $\omega$ scans were performed for 63 complete sets of lattice equivalent reflections. In addition, 3D $Q$ scans were obtained for three sharp reflections (00-10, 4-5-2, 41-2). Fig. S4.1, Fig. S4.2 and Fig. S4.3 show three sets of $\omega$ scans, while Fig. S4.4 shows one $Q$ scan.

Confirming preliminary findings (Gregorkiewitz et al., 1991), reflections with parity $h-k=3 n$ appear systematically sharp $\left(0.008^{\circ}<F W H M<0.012^{\circ}\right)$ with approximately Gaussian shape while the remainder $(h-$ $k \neq 3 n$ ) shows $F W H M \simeq 0.035^{\circ}$ with a more Lorentzian shape.

With the available data (about 620 reflections in 63 complete sets, about half of which had a signal/background ratio < 3), no obvious violations of Laue symmetry $P 6 / \mathrm{mmm}$ could be observed. 

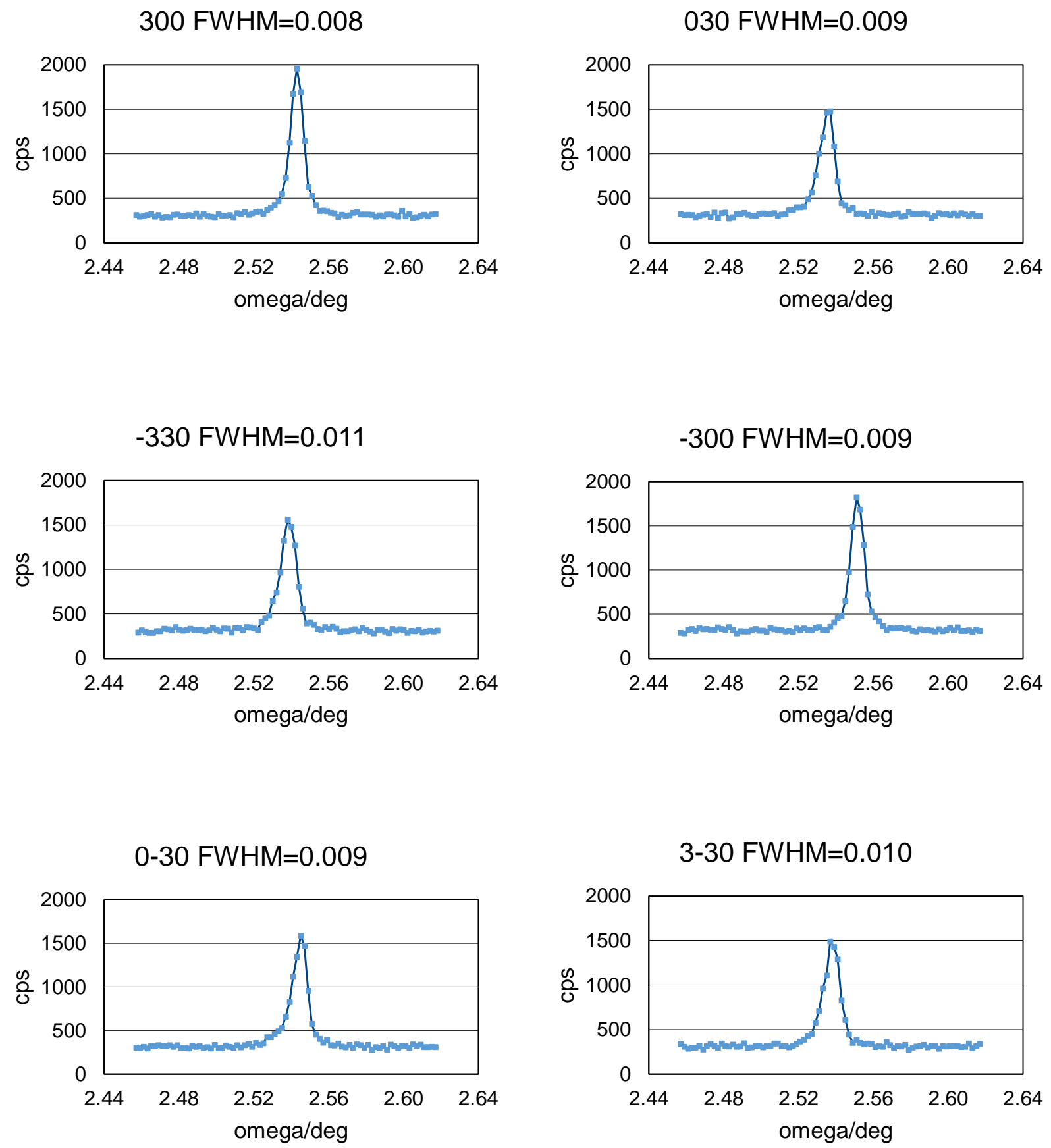

Figure S4.1. Scans obtained in the 6 lattice equivalent positions of reflection 300 ( $h-k=3 n$; Friedel equivalents included). 
30-2 FWHM=0.011

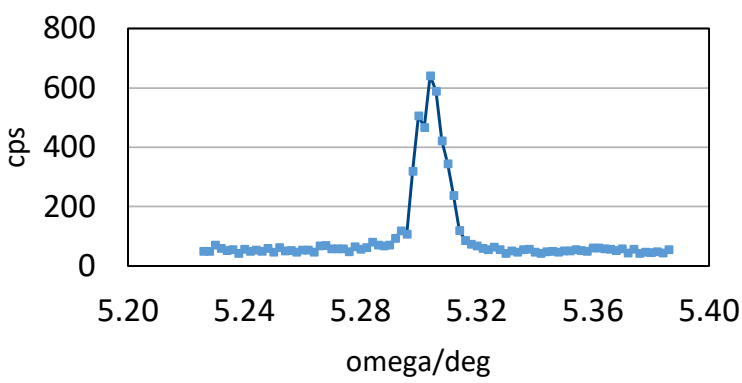

$-33-2$ FWHM=0.011

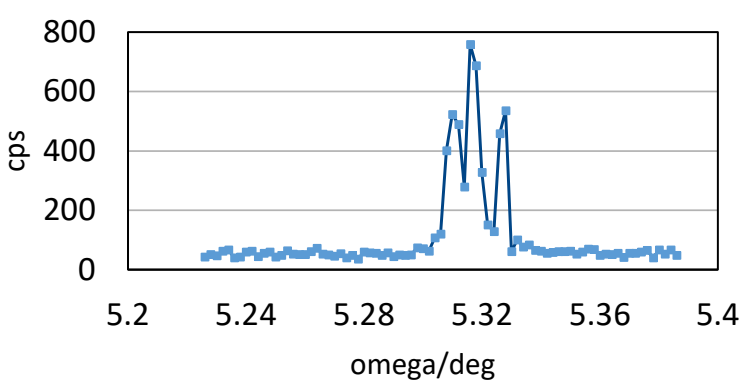

0-3-2 FWHM=0.010

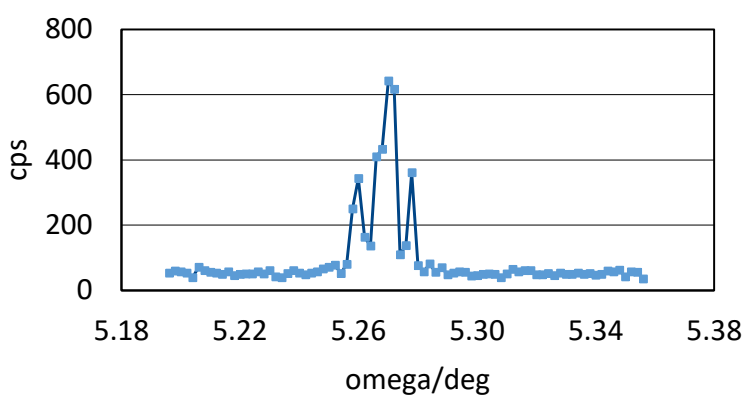

03-2 FWHM=0.012

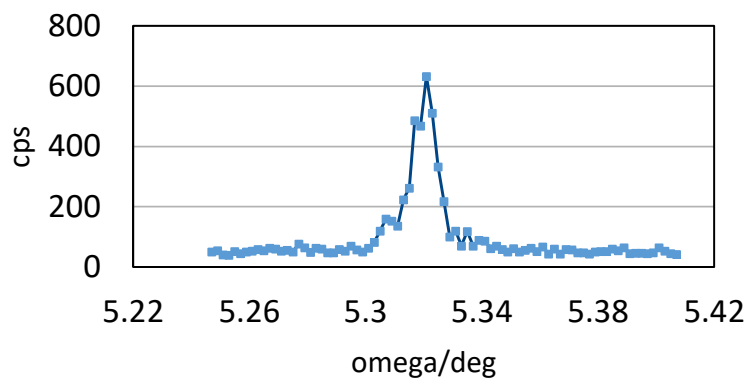

$-30-2$ FWHM=0.009

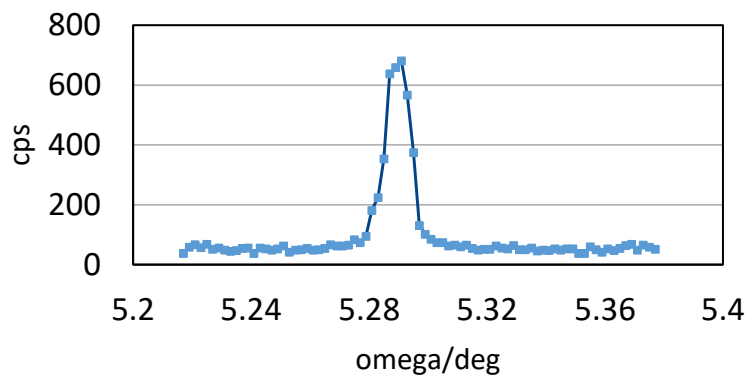

3-3-2 FWHM=0.011

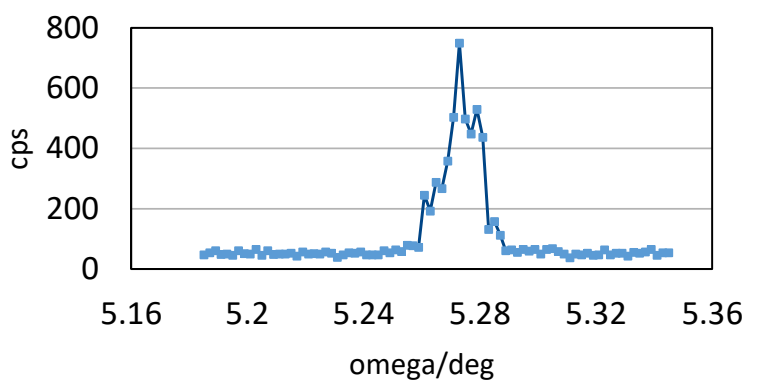

Figure S4.2. Scans obtained in the 6 lattice equivalent positions of reflection 30-2 ( $h-k=3 n$; Friedel equivalents ignored). 

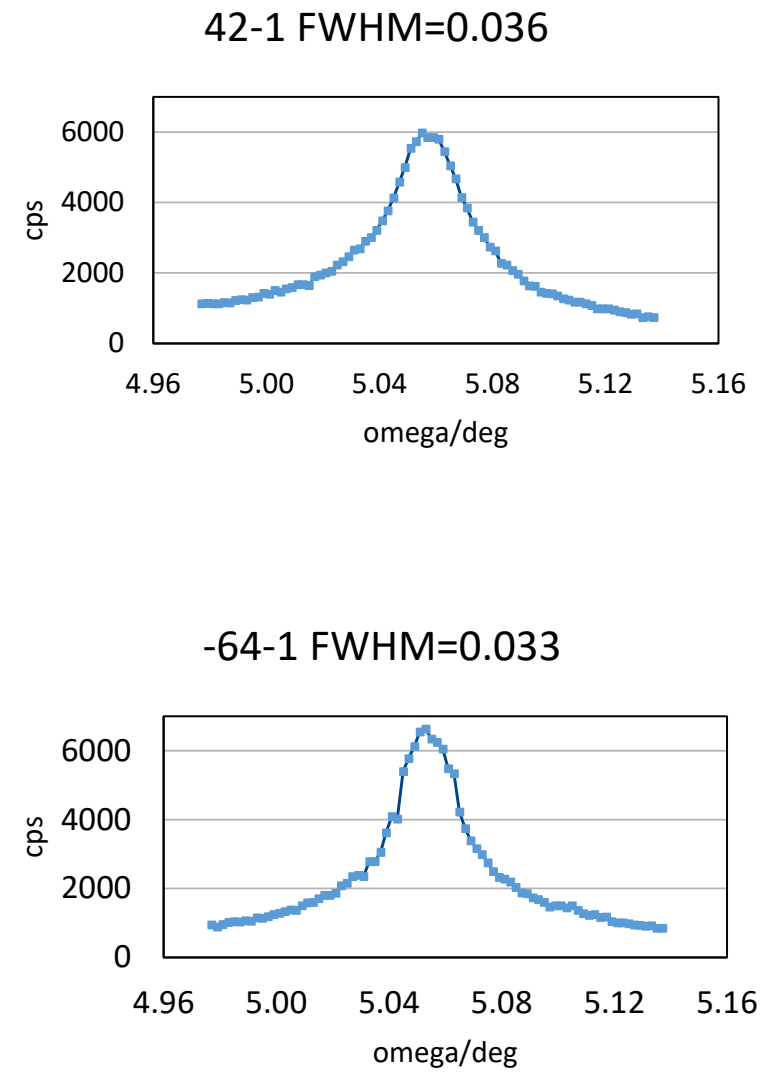

2-6-1 FWHM=0.034

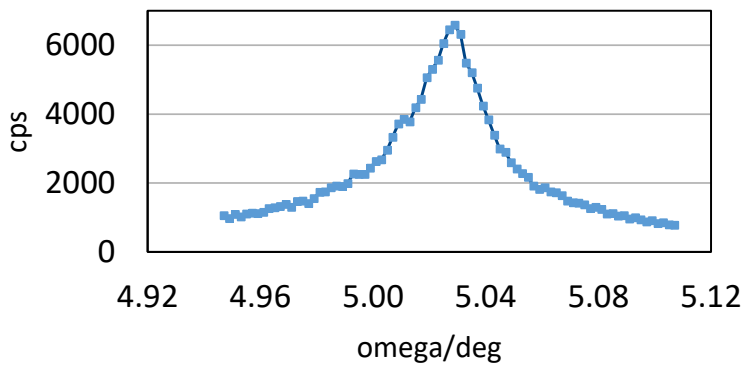

$-26-1$ FWHM=0.035

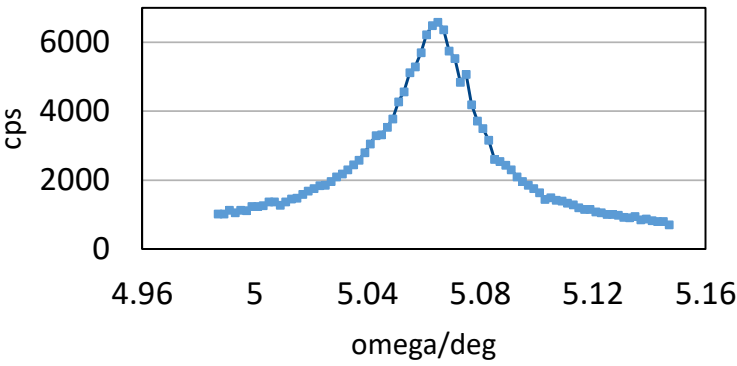

$-4-2-1$ FWHM=0.035

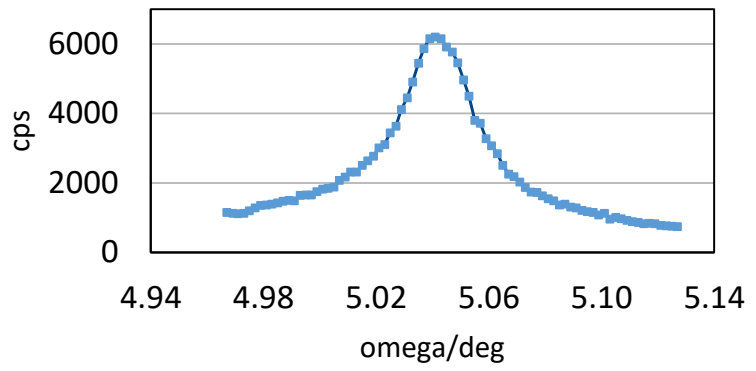

6-4-1 FWHM=0.034

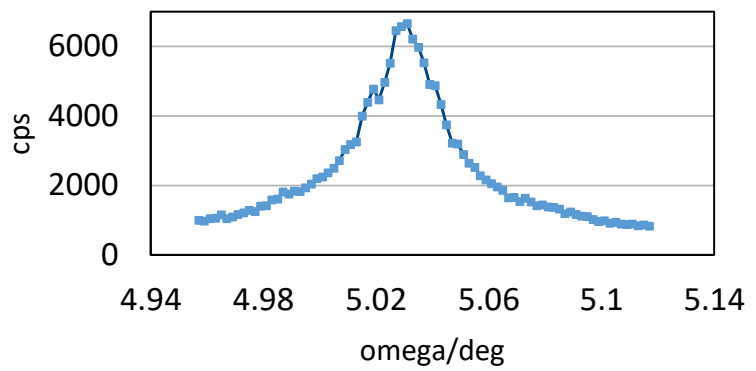

(Figure S4.3 continues in the next page) 


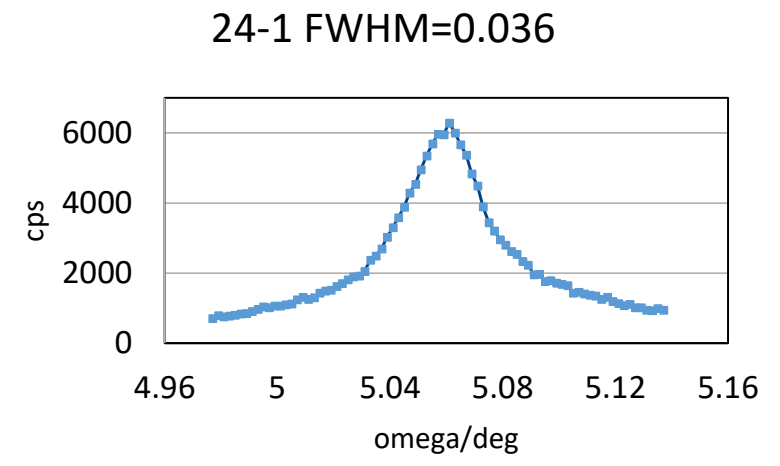

$-62-1$ FWHM=0.035

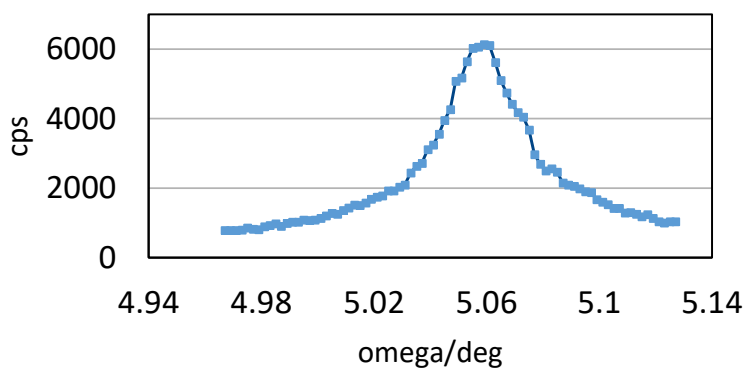

4-6-1 FWHM=0.032

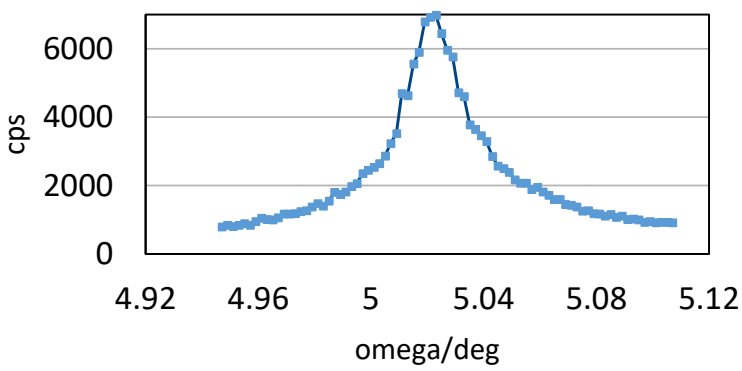

$-46-1$ FWHM=0.031

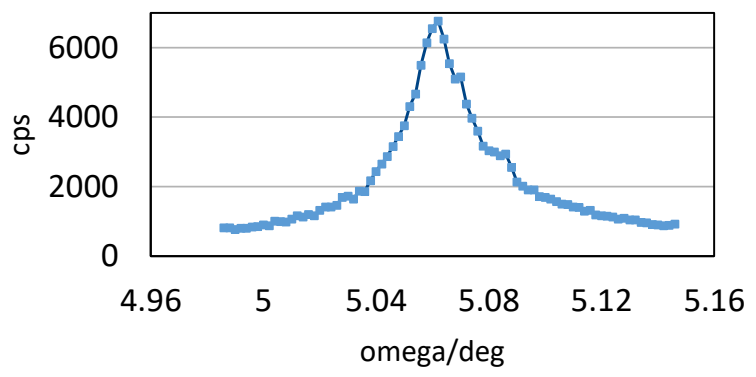

$-2-4-1$ FWHM=0.033

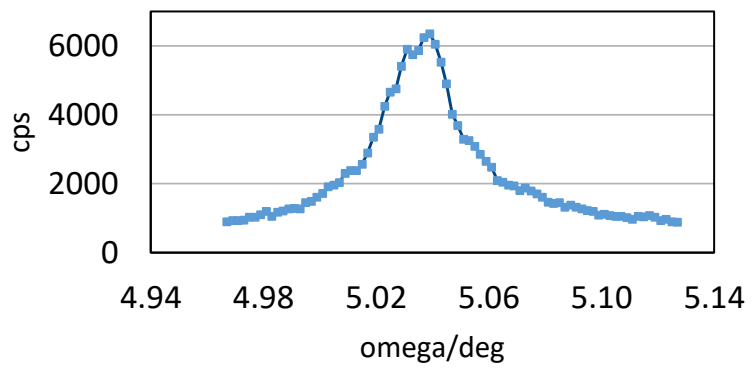

6-2-1 FWHM=0.036

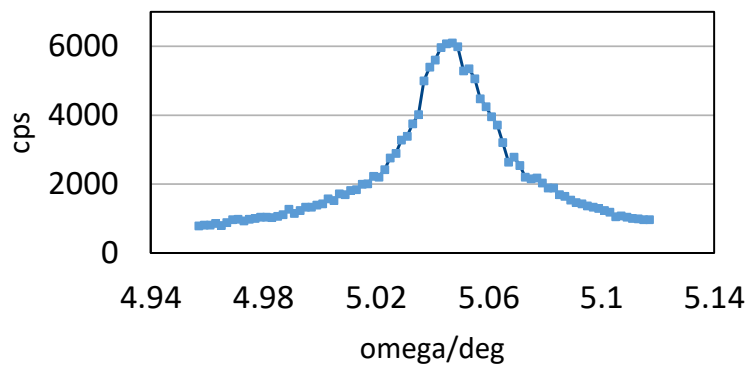

Figure S4.3. Scans obtained in the 12 lattice equivalent positions of reflection $42-1(h-k \neq 3 n$; Friedel equivalents ignored). 


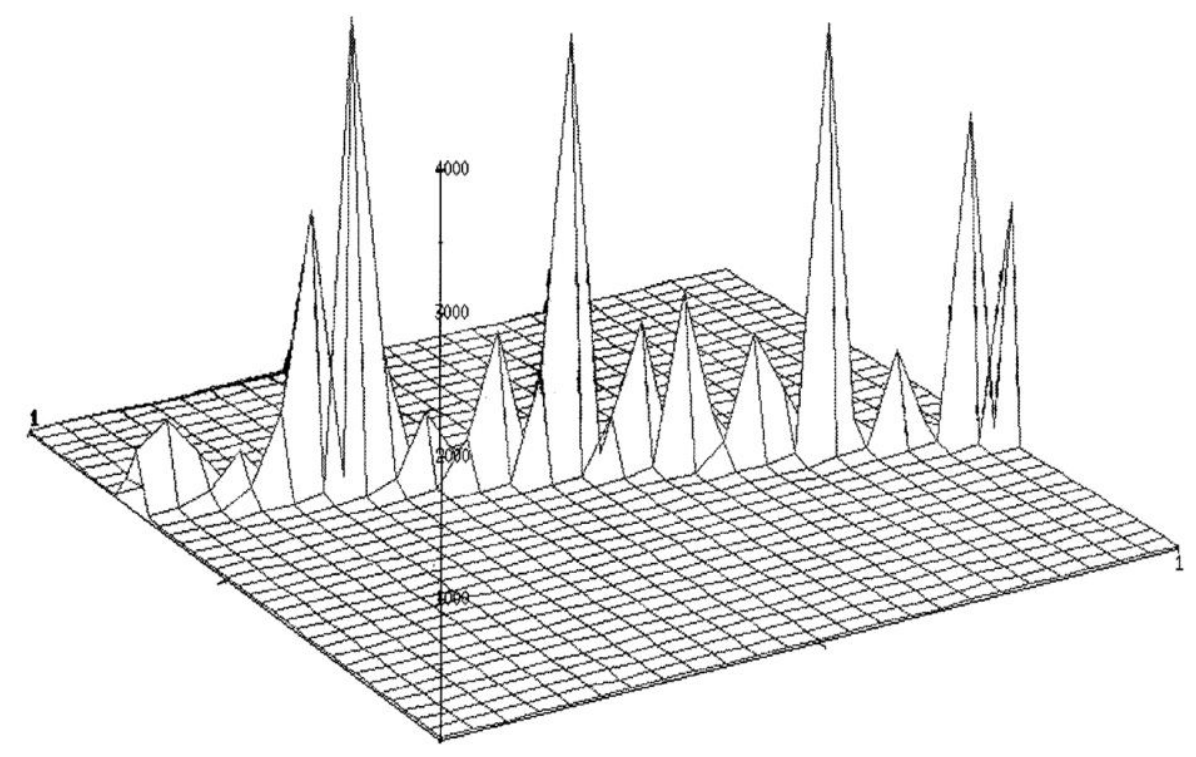

Figure S4.4. 3D representation of one layer of $Q$ scan for reflection 4-5-2 $(h-k=3 n$; step size: $\delta h=\delta k$ $\left.\sim 0.005 \AA^{-1}\right)$. 


\section{References}

Gregorkiewitz, M., Artioli, G., Bessière, M. \& Elkaim, E. (1991). International Meeting on Synchrotron Radiation in Crystallography, Trieste, Italy, p. 36. 


\section{S5. Crystal data, atom parameters, T-O and K-O interatomic distances}

Table S5.1. Experimental details and crystallographic data related with the kaliophilite model obtained by $3 \mathrm{D}$ ED in space group $P 3 c 1$.

\section{Crystal data}

Chemical formula

Crystal system, space group

Temperature (K)

$a, c(\AA)$

Z

Radiation type, $\lambda(\AA)$

Type

Tilt range $\left({ }^{\circ}\right)$, tilt step $\left(^{\circ}\right)$
$\mathrm{Al}_{9} \mathrm{~K}_{9} \mathrm{Si}_{9} \mathrm{O}_{36}$

Trigonal, $P 3 c 1$

293

$27.1(5), 8.6(2)$

6

\section{Data collection}

Electrons, 0.0335

Step-wise with precessing beam $\left(1^{\circ}\right.$ precession angle $)$

115,1

\section{$A b$ initio structure determination}

No. of measured, independent reflections

11743,1085

$R_{\text {int }}(F)$

0.266

$(\sin \theta / \lambda)_{\max }\left(\AA^{-1}\right)$

1.00

Reflection completeness at $1.00 \AA^{-1}(\%)$

99

Computer programs

SIR2014 (Burla et al., 2015)

\section{Structure refinement}

No. of measured, independent, and observed

$11019,3754,2764$

$[I>2 \sigma(I)]$ reflections

$R\left[F^{2}>4 \sigma\left(F^{2}\right)\right], w R\left(F^{2}\right)$, Goof

$0.350,0.641,2.00$

No. of parameters

193

Computer programs 
Table S5.2. Experimental details and crystallographic data for the single crystal X-ray diffraction refinement of kaliophilite from Pollena (Vesuvius area, Naples, Italy) in space group P3.

\section{Crystal data}

Chemical formula

Crystal system, space group

Temperature (K)

$a, c(\AA)$

$V\left(\AA^{3}\right)$

Radiation type

$\mu\left(\mathrm{mm}^{-1}\right)$

Crystal size $(\mathrm{mm})$

Diffractometer

Absorption correction

$T_{\min }, T_{\max }$

No. of measured, independent, and observed

$[I>2 \sigma(I)]$ reflections

$R_{\text {int }}$

$(\sin \theta / \lambda)_{\max }\left(\AA^{-1}\right)$

$R\left[F^{2}>2 \sigma\left(F^{2}\right)\right], w R\left(F^{2}\right), S$

No. of reflections

No. of parameters

No. of restraints

Weight scheme

$\Delta \rho_{\max }, \Delta \rho_{\min }\left(\mathrm{e} \AA^{-3}\right)$

Absolute structure

Twin law, twin fraction

Computer programs
$\mathrm{K}_{8.22} \mathrm{Na}_{0.78} \mathrm{Si}_{9} \mathrm{Al}_{9} \mathrm{O}_{36}$

Trigonal, $P 3$

293

27.0597 (16), 8.5587 (6)

$5427.3(7)$

$\mathrm{MoK} \alpha$

1.68

$0.1 \times 0.15 \times 0.30$

\section{Data collection}

Bruker Smart Breeze diffractometer equipped with an air-cooled CCD detector

Multi-scan

$0.523,0.747$

$127892,37707,33165$

0.061

0.859

\section{Structure refinement}

$0.0768,0.1822,1.02$

37707

589

1

$w=1 /\left[\sigma^{2}\left(F_{\mathrm{o}}^{2}\right)+(0.0519 P)^{2}+59.5879 P\right]$ where $P=\left(F_{\mathrm{o}}^{2}+2 F_{\mathrm{c}}{ }^{2}\right) / 3$

$2.29,-1.54$

Twinning involves inversion, so Flack parameter cannot be determined

(1-10), $\alpha=0.45$

SHELXL (Sheldrick, 2015) 

Table S5.3. Atomic coordinates $\left(\times 10^{4}\right)$ and equivalent isotropic displacement parameters $\left(\AA^{2} \times 10^{3}\right)$ for kaliophilite. $U_{\text {eq }}$ is used only for $\mathrm{K}$ atoms when no K-Na substitution occurs (site $4 \mathrm{~B}$ ) and is defined as one third of the trace of the orthogonalized $U_{\mathrm{ij}}$ tensor.

\begin{tabular}{|c|c|c|c|c|c|c|c|c|c|}
\hline Atom & $x$ & $y$ & $z$ & $U_{\text {eq }} / U_{\text {iso }}$ & Atom & $x$ & $y$ & $z$ & $U_{\text {eq }} / U_{\text {iso }}$ \\
\hline Sil & 992(1) & $1114(1)$ & $4140(3)$ & $7(1)$ & SilB & $-115(1)$ & 1079(1) & $-857(3)$ & $7(1)$ \\
\hline $\mathrm{Si} 2$ & $5574(1)$ & $3332(1)$ & $4128(3)$ & $5(1)$ & $\mathrm{Si} 2 \mathrm{~B}$ & 5581(1) & $2254(1)$ & $-879(3)$ & $4(1)$ \\
\hline $\mathrm{Si} 3$ & $3344(1)$ & $5575(1)$ & $5304(3)$ & $8(1)$ & $\mathrm{Si3B}$ & $2237(1)$ & $5557(1)$ & $302(3)$ & $9(1)$ \\
\hline $\mathrm{Si} 4$ & 2231(1) & 2104(1) & $-875(3)$ & $7(1)$ & Si4B & 2397(1) & $285(1)$ & $4071(3)$ & $7(1)$ \\
\hline $\mathrm{Si} 5$ & $3414(1)$ & $2278(1)$ & $4136(3)$ & $6(1)$ & Si5B & $3572(1)$ & $1267(1)$ & $-896(3)$ & $6(1)$ \\
\hline Si6 & $4496(1)$ & $3435(1)$ & 296(3) & $8(1)$ & Si6B & $4558(1)$ & $978(1)$ & $5284(3)$ & $9(1)$ \\
\hline Si7 & 4361(1) & $4477(1)$ & $5296(3)$ & $8(1)$ & Si7B & $238(1)$ & $4612(1)$ & $305(3)$ & $8(1)$ \\
\hline $\mathrm{Si} 8$ & $3153(1)$ & $4329(1)$ & $-803(3)$ & $6(1)$ & Si8B & 1194(1) & $4285(1)$ & $4187(3)$ & $5(1)$ \\
\hline Si9 & $2110(1)$ & $3130(1)$ & $4173(3)$ & $8(1)$ & Si9B & $954(1)$ & $3044(1)$ & $-880(3)$ & $7(1)$ \\
\hline Al1 & 992(1) & $1115(1)$ & $345(3)$ & $4(1)$ & Al1B & $-116(1)$ & 1081(1) & $5357(3)$ & $5(1)$ \\
\hline Al2 & $5572(1)$ & $3334(1)$ & $351(3)$ & $3(1)$ & $\mathrm{A} 12 \mathrm{~B}$ & $5580(1)$ & 2254(1) & $5352(3)$ & $2(1)$ \\
\hline $\mathrm{A} 13$ & $3343(1)$ & $5573(1)$ & $-905(3)$ & $6(1)$ & A13B & $2232(1)$ & $5557(1)$ & $4098(3)$ & $7(1)$ \\
\hline A14 & $2228(1)$ & $2100(1)$ & $5343(3)$ & $3(1)$ & A14B & 2398(1) & $288(1)$ & $399(3)$ & $3(1)$ \\
\hline A15 & $3414(1)$ & $2278(1)$ & $341(3)$ & $4(1)$ & A15B & $3576(1)$ & $1273(1)$ & $5360(3)$ & $3(1)$ \\
\hline Al6 & $4495(1)$ & $3436(1)$ & $4119(3)$ & $6(1)$ & Al6B & $4562(1)$ & $978(1)$ & $-860(3)$ & $6(1)$ \\
\hline Al7 & $4359(1)$ & $4476(1)$ & $-890(3)$ & $7(1)$ & Al7B & $242(1)$ & $4619(1)$ & 4112(3) & $6(1)$ \\
\hline A18 & $3147(1)$ & $4325(1)$ & $5315(3)$ & $4(1)$ & A18B & $1197(1)$ & $4284(1)$ & $318(3)$ & $3(1)$ \\
\hline A19 & 2102(1) & $3122(1)$ & $321(3)$ & $6(1)$ & A19B & 951(1) & $3040(1)$ & $5374(3)$ & $3(1)$ \\
\hline $\mathrm{Na} 1$ & 0 & 0 & 2281(8) & $6(1)$ & K1B & 0 & 0 & $7245(6)$ & $18(1)$ \\
\hline K2 & 6667 & 3333 & $2233(6)$ & $20(1)$ & K2B & 6667 & 3333 & $7230(5)$ & $13(1)$ \\
\hline K3 & 3333 & 6667 & $2203(5)$ & $12(1)$ & K3B & 3333 & 6667 & $7171(7)$ & $26(1)$ \\
\hline \multirow[t]{2}{*}{ K4 } & 2197(1) & $1255(1)$ & $2240(3)$ & $14(1)$ & K4B & $2405(1)$ & $1107(1)$ & $7240(3)$ & $15(1)$ \\
\hline & & & & & $\mathrm{Na} 4 \mathrm{~B}$ & $2255(10)$ & $1209(9)$ & $7240(30)$ & $15(1)$ \\
\hline K5 & $4521(1)$ & 1944(1) & $2227(3)$ & $16(1)$ & K5B & $4537(1)$ & 2571(1) & $7220(3)$ & $18(1)$ \\
\hline K6 & $5586(1)$ & $4546(1)$ & 2217(3) & 12(1) & K6B & 5741(1) & $1140(1)$ & $7209(3)$ & $19(1)$ \\
\hline
\end{tabular}




\begin{tabular}{|c|c|c|c|c|c|c|c|c|c|}
\hline K7 & 4090(1) & $5296(1)$ & $2223(3)$ & $16(1)$ & K7B & $1205(1)$ & $5258(1)$ & $7228(3)$ & $13(1)$ \\
\hline K8 & $2375(1)$ & $4455(1)$ & $2241(3)$ & $17(1)$ & $\mathrm{K} 8 \mathrm{~B}$ & $2080(1)$ & $4356(1)$ & $7238(3)$ & $17(1)$ \\
\hline K9 & $855(1)$ & $2138(1)$ & $2243(3)$ & $16(1)$ & $\mathrm{Na9B}$ & $1125(2)$ & $2069(2)$ & $7256(5)$ & $8(1)$ \\
\hline K10 & $3326(1)$ & $3408(1)$ & $2222(3)$ & $19(1)$ & K10B & $108(2)$ & $3429(2)$ & $7230(4)$ & $36(1)$ \\
\hline K11 & $3310(1)$ & $3210(1)$ & $7230(3)$ & $13(1)$ & K11B & $-152(1)$ & $3308(1)$ & $2221(4)$ & $24(1)$ \\
\hline $\mathrm{O} 1$ & $821(4)$ & $1046(4)$ & $2256(12)$ & $24(2)$ & O1B & $-218(5)$ & $1133(5)$ & $7269(13)$ & $29(2)$ \\
\hline $\mathrm{O} 2$ & 5691(3) & $3533(3)$ & $2247(7)$ & $6(1)$ & $\mathrm{O} 2 \mathrm{~B}$ & $5487(3)$ & $2360(3)$ & $7228(8)$ & $8(1)$ \\
\hline $\mathrm{O} 3$ & $3127(4)$ & $5490(4)$ & $7197(11)$ & $23(2)$ & $\mathrm{O} 3 \mathrm{~B}$ & 2111(4) & $5638(4)$ & $2201(12)$ & $22(2)$ \\
\hline $\mathrm{O} 4$ & 2040(3) & 2010(3) & $7251(9)$ & $14(1)$ & O4B & $2626(3)$ & $504(3)$ & $2247(8)$ & $10(1)$ \\
\hline O5 & $3264(3)$ & 2314(3) & $2244(9)$ & $13(1)$ & O5B & $3737(3)$ & $1468(3)$ & $7230(8)$ & $11(1)$ \\
\hline O6 & 4481(3) & $3312(4)$ & $2197(10)$ & $17(1)$ & O6B & 4492(4) & $815(4)$ & $7160(10)$ & $19(2)$ \\
\hline $\mathrm{O} 7$ & $4335(4)$ & $4320(4)$ & $7200(11)$ & $19(2)$ & O7B & $387(3)$ & $4785(3)$ & $2200(9)$ & $14(1)$ \\
\hline $\mathrm{O} 8$ & 3121(3) & $4296(3)$ & $7272(9)$ & $14(1)$ & O8B & $1165(3)$ & 4164(3) & $2267(8)$ & $9(1)$ \\
\hline O9 & 2014(4) & $3044(4)$ & $2259(11)$ & $23(2)$ & O9B & $758(3)$ & 2862(3) & $7271(10)$ & $15(1)$ \\
\hline $\mathrm{O} 10$ & $614(3)$ & $1333(3)$ & $5126(9)$ & $15(1)$ & O10B & $581(3)$ & $1327(3)$ & $-655(8)$ & $11(1)$ \\
\hline O11 & $864(4)$ & 494(4) & $4718(11)$ & $19(2)$ & O11B & $876(3)$ & $456(4)$ & $-301(10)$ & $15(1)$ \\
\hline O12 & $3713(3)$ & $1878(3)$ & $4334(8)$ & $10(1)$ & $\mathrm{O} 12 \mathrm{~B}$ & $3710(3)$ & $1846(3)$ & 123(9) & $13(1)$ \\
\hline $\mathrm{O} 13$ & 5381(3) & 2649(3) & $4238(8)$ & $13(1)$ & O13B & $5384(3)$ & $2625(3)$ & $211(8)$ & $10(1)$ \\
\hline O14 & $6179(3)$ & $3718(3)$ & $5114(8)$ & 11(1) & O14B & $6248(3)$ & $2455(3)$ & $-632(8)$ & $9(1)$ \\
\hline O15 & 4769(3) & $5177(3)$ & $5075(8)$ & $10(1)$ & O15B & $410(3)$ & $5211(3)$ & $-626(9)$ & $15(1)$ \\
\hline O16 & 4013(4) & $6054(4)$ & $5118(10)$ & $18(2)$ & O16B & 2047(4) & $5958(4)$ & $-708(10)$ & $17(1)$ \\
\hline O17 & 2945(4) & $5776(4)$ & $4362(11)$ & $22(2)$ & O17B & $2900(3)$ & $5769(3)$ & $50(9)$ & $14(1)$ \\
\hline $\mathrm{O} 18$ & $1467(4)$ & 2871(3) & 4903(9) & $16(1)$ & O18B & $1435(3)$ & $2858(3)$ & $-438(9)$ & $14(1)$ \\
\hline O19 & $1685(4)$ & $1632(4)$ & $206(10)$ & $16(1)$ & O19B & $1815(4)$ & $322(4)$ & $4358(10)$ & $20(2)$ \\
\hline $\mathrm{O} 20$ & $1667(3)$ & $1606(3)$ & $4300(9)$ & $13(1)$ & $\mathrm{O} 20 \mathrm{~B}$ & $1780(4)$ & $314(4)$ & $134(10)$ & $18(1)$ \\
\hline $\mathrm{O} 21$ & $5043(3)$ & $3418(3)$ & $-490(8)$ & $12(1)$ & $\mathrm{O} 21 \mathrm{~B}$ & $5168(3)$ & $1540(3)$ & $4854(9)$ & $15(1)$ \\
\hline $\mathrm{O} 22$ & $5062(3)$ & $3423(3)$ & 4943(8) & $11(1)$ & $\mathrm{O} 22 \mathrm{~B}$ & $5197(3)$ & 1571(3) & $-398(9)$ & $14(1)$ \\
\hline $\mathrm{O} 23$ & $3224(3)$ & $4943(3)$ & $-130(8)$ & $8(1)$ & $\mathrm{O} 23 \mathrm{~B}$ & 1801(3) & $4861(3)$ & 4697(8) & $10(1)$ \\
\hline
\end{tabular}




$\begin{array}{llllllllll}\text { O24 } & 3224(3) & 4960(3) & 4603(8) & 9(1) & \text { O24B } & 1833(3) & 4892(3) & -233(8) & 12(1) \\ \text { O25 } & 2772(3) & 1992(3) & -668(9) & 13(1) & \text { O25B } & 2890(4) & 742(3) & 5290(10) & 17(1) \\ \text { O26 } & 2800(3) & 1996(3) & 5132(8) & 9(1) & \text { O26B } & 2911(4) & 762(4) & -826(10) & 17(1) \\ \text { O27 } & 3875(3) & 2968(3) & -433(9) & 13(1) & \text { O27B } & 4012(3) & 1032(3) & 4623(9) & 15(1) \\ \text { O28 } & 3860(3) & 2924(3) & 4856(8) & 9(1) & \text { O28B } & 3988(3) & 1031(3) & -167(9) & 14(1) \\ \text { O29 } & 4629(4) & 4102(4) & 15(10) & 17(1) & \text { O29B } & 4554(3) & 459(3) & 4272(8) & 13(1) \\ \text { O30 } & 4627(3) & 4126(3) & 4391(8) & 10(1) & \text { O30B } & 4554(3) & 431(3) & 159(9) & 14(1) \\ \text { O31 } & 3667(3) & 4237(3) & -173(9) & 12(1) & \text { O31B } & 655(3) & 4350(3) & 4825(9) & 13(1) \\ \text { O32 } & 3711(3) & 4261(3) & 4581(7) & 6(1) & \text { O32B } & 630(3) & 4349(3) & -368(8) & 9(1) \\ \text { O33 } & 2542(3) & 3841(3) & -117(9) & 15(1) & \text { O33B } & 1193(3) & 3764(3) & 5168(8) & 13(1) \\ \text { O34 } & 2514(3) & 3808(3) & 4549(8) & 10(1) & \text { O34B } & 1193(3) & 3737(3) & -680(7) & 7(1) \\ \text { O35 } & 2444(3) & 2767(3) & -331(9) & 15(1) & \text { O35B } & 370(4) & 2644(4) & 4230(10) & 18(1) \\ \text { O36 } & 2427(3) & 2795(3) & 4848(9) & 14(1) & \text { O36B } & 400(4) & 2687(4) & 221(9) & 16(1)\end{array}$


Table S5.4. Bond lengths $(\AA)$ for the framework of kaliophilite.

\begin{tabular}{|c|c|c|c|c|c|c|c|c|}
\hline \multirow[t]{5}{*}{ Sil } & O11 & $1.611(9)$ & $\mathrm{Si} 2$ & $\mathrm{O} 13$ & $1.654(8)$ & $\mathrm{Si} 3$ & O16 & $1.623(9)$ \\
\hline & $\mathrm{O} 20$ & $1.641(8)$ & & $\mathrm{O} 14$ & $1.666(8)$ & & $\mathrm{O} 24$ & $1.640(7)$ \\
\hline & $\mathrm{O} 10$ & $1.650(8)$ & & $\mathrm{O} 2$ & $1.674(7)$ & & $\mathrm{O} 17$ & $1.643(10)$ \\
\hline & $\mathrm{O} 1$ & $1.663(10)$ & & $\mathrm{O} 22$ & $1.678(8)$ & & $\mathrm{O} 3$ & $1.700(10)$ \\
\hline & average & $1.641(22)$ & & average & $1.668(11)$ & & average & $1.652(34)$ \\
\hline \multirow[t]{5}{*}{$\mathrm{Si} 4$} & $\mathrm{O} 25$ & $1.646(8)$ & $\mathrm{Si} 5$ & $\mathrm{O} 12$ & $1.651(8)$ & Si6 & $\mathrm{O} 27$ & $1.639(8)$ \\
\hline & $\mathrm{O} 35$ & $1.654(9)$ & & $\mathrm{O} 28$ & $1.668(7)$ & & $\mathrm{O} 21$ & $1.647(8)$ \\
\hline & O4\#5 & $1.665(9)$ & & $\mathrm{O} 26$ & $1.673(7)$ & & O6 & $1.658(9)$ \\
\hline & O19 & $1.668(9)$ & & O5 & $1.685(8)$ & & O29 & $1.673(9)$ \\
\hline & average & $1.658(10)$ & & average & $1.669(14)$ & & average & $1.654(15)$ \\
\hline \multirow[t]{5}{*}{$\mathrm{Si7}$} & $\mathrm{O} 30$ & $1.643(7)$ & $\mathrm{Si} 8$ & O31 & $1.623(8)$ & $\mathrm{Si} 9$ & $\mathrm{O} 34$ & $1.630(8)$ \\
\hline & $\mathrm{O} 15$ & $1.660(8)$ & & $\mathrm{O} 33$ & $1.624(8)$ & & $\mathrm{O} 36$ & $1.632(8)$ \\
\hline & $\mathrm{O} 32$ & $1.667(7)$ & & O8\#5 & $1.649(8)$ & & O18 & $1.639(9)$ \\
\hline & $\mathrm{O} 7$ & $1.676(9)$ & & $\mathrm{O} 23$ & $1.678(7)$ & & O9 & $1.657(10)$ \\
\hline & average & $1.662(14)$ & & average & $1.644(26)$ & & average & $1.640(12)$ \\
\hline \multirow[t]{5}{*}{ Si1B } & O11B\#1 & $1.615(9)$ & $\mathrm{Si} 2 \mathrm{~B}$ & O14B & $1.617(7)$ & $\mathrm{Si3B}$ & O17B & $1.602(9)$ \\
\hline & $\mathrm{O} 20 \mathrm{~B} \# 1$ & $1.635(9)$ & & O13B & $1.643(7)$ & & O24B & $1.636(8)$ \\
\hline & $\mathrm{O} 1 \mathrm{~B} \# 5$ & $1.647(11)$ & & $\mathrm{O} 22 \mathrm{~B}$ & $1.655(8)$ & & O16B & $1.656(9)$ \\
\hline & O10B & $1.662(8)$ & & $\mathrm{O} 2 \mathrm{~B} \# 5$ & $1.687(7)$ & & $\mathrm{O} 3 \mathrm{~B}$ & $1.697(10)$ \\
\hline & average & $1.640(20)$ & & average & $1.651(29)$ & & average & $1.648(40)$ \\
\hline \multirow[t]{5}{*}{ Si4B } & O35B\#9 & $1.636(9)$ & $\mathrm{Si} 5 \mathrm{~B}$ & O26B & $1.620(9)$ & Si6B & $\mathrm{O} 21 \mathrm{~B}$ & $1.632(9)$ \\
\hline & O19B & $1.647(9)$ & & $\mathrm{O} 28 \mathrm{~B}$ & $1.667(8)$ & & O29B & $1.646(8)$ \\
\hline & O25B & $1.657(9)$ & & O12B & $1.666(8)$ & & O6B & $1.651(9)$ \\
\hline & O4B & $1.675(7)$ & & $\mathrm{O} 5 \mathrm{~B} \# 5$ & $1.680(7)$ & & O27B & $1.655(8)$ \\
\hline & average & $1.654(17)$ & & average & $1.658(26)$ & & average & $1.646(10)$ \\
\hline \multirow[t]{2}{*}{ Si7B } & O30B\#1 & $1.628(8)$ & $\mathrm{Si} 8 \mathrm{~B}$ & O33B & $1.638(8)$ & Si9B & O36B & $1.618(9)$ \\
\hline & O32B & $1.647(7)$ & & O31B & $1.649(8)$ & & O18B & $1.657(9)$ \\
\hline
\end{tabular}




\begin{tabular}{|c|c|c|c|c|c|c|c|c|}
\hline & O15B & $1.652(8)$ & & O23B & $1.661(7)$ & & O34B & $1.658(7)$ \\
\hline & O7B & $1.680(8)$ & & $\mathrm{O} 8 \mathrm{~B}$ & $1.669(7)$ & & $\mathrm{O} 9 \mathrm{~B} \# 5$ & $1.664(8)$ \\
\hline & average & $1.652(21)$ & & average & $1.654(14)$ & & average & $1.649(21)$ \\
\hline \multirow[t]{5}{*}{ Al1 } & $\mathrm{O} 1$ & $1.685(10)$ & $\mathrm{A} 12$ & $\mathrm{O} 2$ & $1.690(7)$ & $\mathrm{A} 13$ & $\mathrm{O} 23$ & $1.703(7)$ \\
\hline & O19 & $1.692(9)$ & & $\mathrm{O} 21$ & $1.717(8)$ & & $\mathrm{O} 3 \# 5$ & $1.703(10)$ \\
\hline & O10B & $1.713(8)$ & & O13B & $1.725(7)$ & & O16B\#3 & $1.709(9)$ \\
\hline & O11B & $1.740(9)$ & & O14B\#2 & $1.731(7)$ & & O17B & $1.738(9)$ \\
\hline & average & $1.708(25)$ & & average & $1.716(18)$ & & average & $1.713(17)$ \\
\hline \multirow[t]{5}{*}{ Al4 } & $\mathrm{O} 20$ & $1.692(8)$ & A15 & O5 & $1.693(8)$ & Al6 & O6 & $1.675(9)$ \\
\hline & $\mathrm{O} 4$ & $1.691(9)$ & & O12B & $1.725(8)$ & & $\mathrm{O} 28$ & $1.700(7)$ \\
\hline & $\mathrm{O} 26$ & $1.717(7)$ & & $\mathrm{O} 25$ & $1.735(8)$ & & $\mathrm{O} 22$ & $1.704(8)$ \\
\hline & $\mathrm{O} 36$ & $1.730(8)$ & & $\mathrm{O} 27$ & $1.775(8)$ & & $\mathrm{O} 30$ & $1.734(8)$ \\
\hline & average & $1.708(19)$ & & average & $1.732(34)$ & & average & $1.703(24)$ \\
\hline \multirow[t]{5}{*}{$\mathrm{A} 17$} & $\mathrm{O} 7 \# 5$ & $1.681(9)$ & $\mathrm{A} 18$ & O8 & $1.677(8)$ & A19 & O9 & $1.674(10)$ \\
\hline & $\mathrm{O} 29$ & $1.703(9)$ & & $\mathrm{O} 34$ & $1.709(8)$ & & O18B & $1.703(8)$ \\
\hline & O15B\#3 & $1.720(9)$ & & $\mathrm{O} 24$ & $1.737(7)$ & & $\mathrm{O} 35$ & $1.726(8)$ \\
\hline & $\mathrm{O} 31$ & $1.756(8)$ & & $\mathrm{O} 32$ & $1.738(7)$ & & $\mathrm{O} 33$ & 1.741(9) \\
\hline & average & $1.715(32)$ & & average & $1.715(29)$ & & average & $1.711(29)$ \\
\hline \multirow[t]{5}{*}{ Al1B } & O1B & $1.677(11)$ & $\mathrm{A} 12 \mathrm{~B}$ & $\mathrm{O} 2 \mathrm{~B}$ & $1.671(7)$ & A13B & $\mathrm{O} 3 \mathrm{~B}$ & $1.692(10)$ \\
\hline & O19B\#1 & $1.705(9)$ & & $\mathrm{O} 21 \mathrm{~B}$ & $1.734(8)$ & & O23B & $1.725(8)$ \\
\hline & $\mathrm{O} 10$ & $1.750(8)$ & & $\mathrm{O} 14$ & $1.704(8)$ & & O16 & $1.725(10)$ \\
\hline & O11 & $1.753(9)$ & & $\mathrm{O} 13$ & $1.706(8)$ & & 017 & $1.716(10)$ \\
\hline & average & $1.721(37)$ & & average & $1.704(26)$ & & average & $1.715(16)$ \\
\hline \multirow[t]{5}{*}{ Al4B } & O4B & $1.692(7)$ & A15B & O5B & $1.674(7)$ & $\mathrm{Al6B}$ & O30B & $1.708(8)$ \\
\hline & $\mathrm{O} 26 \mathrm{~B}$ & $1.701(9)$ & & $\mathrm{O} 25 \mathrm{~B}$ & $1.687(9)$ & & O22B & $1.711(8)$ \\
\hline & $\mathrm{O} 20 \mathrm{~B}$ & $1.724(9)$ & & O27B & $1.730(8)$ & & O28B & $1.735(8)$ \\
\hline & O36B\#9 & $1.736(9)$ & & $\mathrm{O} 12$ & $1.727(7)$ & & O6B\#5 & $1.737(9)$ \\
\hline & average & $1.713(20)$ & & average & $1.704(28)$ & & average & $1.722(15)$ \\
\hline
\end{tabular}




\begin{tabular}{|c|c|c|c|c|c|c|c|c|}
\hline \multirow[t]{5}{*}{ Al7B } & O7B & $1.690(8)$ & Al8B & $\mathrm{O} 8 \mathrm{~B}$ & $1.694(7)$ & A19B & O35B & $1.702(9)$ \\
\hline & O29B\#1 & $1.713(8)$ & & O34B & $1.704(7)$ & & O9B & $1.700(9)$ \\
\hline & O31B & $1.722(8)$ & & O32B & $1.733(8)$ & & O33B & $1.739(8)$ \\
\hline & O15 & $1.696(78)$ & & $\mathrm{O} 24 \mathrm{~B}$ & $1.748(8)$ & & O18 & $1.720(9)$ \\
\hline & average & $1.705(15)$ & & average & $1.720(25)$ & & average & $1.715(18)$ \\
\hline
\end{tabular}

\#1 -y,x-y,z; \#2 -x+y+1,-x+1,z; \#3 -y+1,x-y+1,z; \#4 -y+1,x-y+1,z+1; \#5 x,y,z-1; \#6 -x+y+1,-x+1,z-1; \#7 -y+1,x-y+1,z-1; \#8 x,y,z+1; \#9 -x+y,-x,z; \#10 -y+1,x-y,z; \#11 -x+y,-x+1,z; \#12 -y,x-y,z-1; \#13 $\mathrm{x}+\mathrm{y},-\mathrm{x}, \mathrm{z}-1$; \#14 -y,x-y,z+1; \#15 -x+y,-x,z+1; \#16 -y+1,x-y,z+1; \#17 -x+y+1,-x+1,z+1; \#18 -x+y,$\mathrm{x}+1, \mathrm{z}+1 ; \# 19-\mathrm{y}+1, \mathrm{x}-\mathrm{y}, \mathrm{z}-1$ \#20 -x+y,-x+1,z-1 
Table S5.5 Bond lengths $(\AA)$ for the extra-framework cations in kaliophilite.

\begin{tabular}{|c|c|c|c|c|c|c|c|c|}
\hline \multirow[t]{2}{*}{$\mathrm{Na} 1$} & O1 & $2.580(10) \times 3$ & K1B & O11B & $2.938(9) \times 3$ & & & \\
\hline & O11 & 2.911(10) X 3 & & O1B & $3.398(11) \times 3$ & & & \\
\hline \multirow[t]{3}{*}{ K2 } & $\mathrm{O} 2$ & $2.949(6) \times 3$ & $\mathrm{~K} 2 \mathrm{~B}$ & O14 & $2.734(8) \times 3$ & & & \\
\hline & O14B & $3.201(8) \times 3$ & & O14B & $2.754(7) \times 3$ & & & \\
\hline & $\mathrm{O} 14$ & $3.205(8) \times 3$ & & $\mathrm{O} 2 \mathrm{~B}$ & $2.953(6) \times 3$ & & & \\
\hline \multirow[t]{3}{*}{ K3 } & O17B & $2.797(8) \times 3$ & K3B & $\mathrm{O} 3$ & $2.944(10)$ X 3 & & & \\
\hline & O17 & $2.792(10) \times 3$ & & O17 & $3.188(10) \times 3$ & & & \\
\hline & O3B & $3.079(9) \times 3$ & & O17B & $3.240(9) \times 3$ & & & \\
\hline \multirow[t]{9}{*}{ K4 } & O19 & $2.720(9)$ & K4B & $\mathrm{O} 26 \mathrm{~B}$ & $2.601(9)$ & $\mathrm{Na} 4 \mathrm{~B}$ & O4 & $2.51(2)$ \\
\hline & $\mathrm{O} 20$ & $2.727(8)$ & & $\mathrm{O} 25 \mathrm{~B}$ & $2.604(9)$ & & O1B & $2.57(2)$ \\
\hline & $\mathrm{O} 4 \mathrm{~B}$ & $2.799(7)$ & & O1B & $2.658(11)$ & & $\mathrm{O} 25$ & $2.59(2)$ \\
\hline & O19B & $2.850(9)$ & & $\mathrm{O} 25$ & $2.747(8)$ & & $\mathrm{O} 26$ & $2.61(2)$ \\
\hline & O20B & $2.851(9)$ & & O26 & $2.759(8)$ & & $\mathrm{O} 25 \mathrm{~B}$ & $3.08(3)$ \\
\hline & O5 & $2.877(8)$ & & $\mathrm{O} 4$ & $3.058(9)$ & & $\mathrm{O} 26 \mathrm{~B}$ & $3.08(3)$ \\
\hline & $\mathrm{O} 25$ & $3.081(8)$ & & O19B & $3.124(9)$ & & & \\
\hline & $\mathrm{O} 26$ & $3.089(7)$ & & $\mathrm{O} 20 \mathrm{~B}$ & $3.158(9)$ & & & \\
\hline & & & & O5B & $3.231(8)$ & & & \\
\hline \multirow[t]{10}{*}{ K5 } & O13B & $2.744(7)$ & K5B & O5B & $2.671(7)$ & & & \\
\hline & $\mathrm{O} 13$ & $2.753(8)$ & & $\mathrm{O} 21$ & $2.798(8)$ & & & \\
\hline & O12B & $2.747(8)$ & & $\mathrm{O} 22$ & $2.803(8)$ & & & \\
\hline & $\mathrm{O} 12$ & $2.770(7)$ & & $\mathrm{O} 2 \mathrm{~B}$ & $2.898(7)$ & & & \\
\hline & $\mathrm{O} 27 \mathrm{~B}$ & $2.966(8)$ & & $\mathrm{O} 28$ & $3.183(7)$ & & & \\
\hline & O28B & $2.970(8)$ & & $\mathrm{O} 27$ & $3.213(8)$ & & & \\
\hline & $\mathrm{O} 21 \mathrm{~B}$ & $3.352(8)$ & & $\mathrm{O} 12$ & $3.227(7)$ & & & \\
\hline & $\mathrm{O} 22 \mathrm{~B}$ & $3.355(8)$ & & O12B & $3.262(8)$ & & & \\
\hline & & & & O13 & $3.361(8)$ & & & \\
\hline & & & & O13B & $3.390(7)$ & & & \\
\hline
\end{tabular}




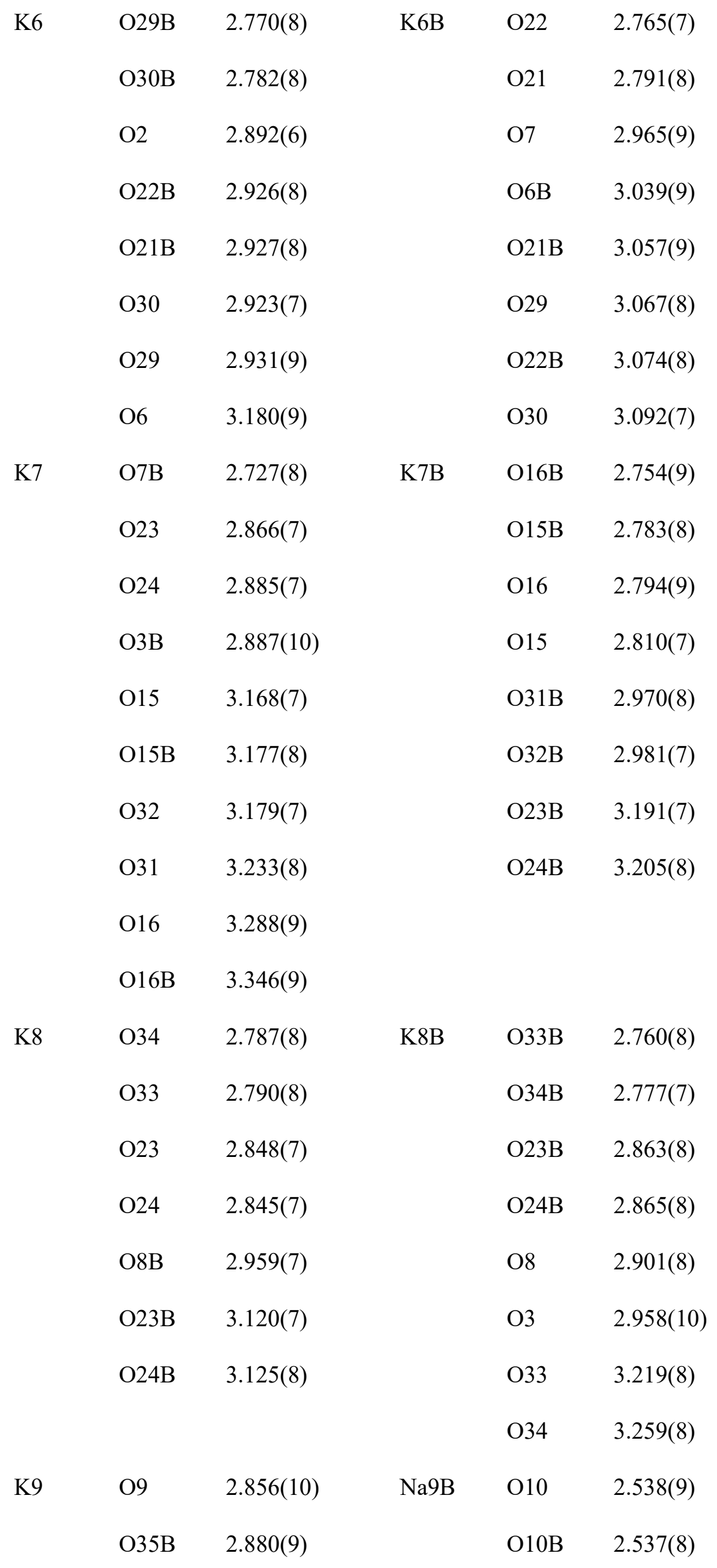




\begin{tabular}{|c|c|c|c|c|c|}
\hline & O1 & $2.909(10)$ & & $\mathrm{O} 4$ & $2.559(9)$ \\
\hline & O18B & $2.910(8)$ & & O18B & $2.716(8)$ \\
\hline & O18 & $2.928(8)$ & & $\mathrm{O} 18$ & $2.760(9)$ \\
\hline & O36B & $2.924(9)$ & & O9B & $2.779(9)$ \\
\hline & $\mathrm{O} 10$ & $3.135(8)$ & & & \\
\hline & O10B & $3.145(8)$ & & & \\
\hline & O20B & $3.289(9)$ & & & \\
\hline & O19B & $3.302(9)$ & & & \\
\hline K10 & $\mathrm{O} 31$ & $2.833(8)$ & K10B & O9B & $2.854(8)$ \\
\hline & $\mathrm{O} 32$ & $2.843(7)$ & & O6B & $2.893(9)$ \\
\hline & O5 & $2.877(8)$ & & O32B & $2.983(8)$ \\
\hline & $\mathrm{O} 35$ & $3.056(9)$ & & O31B & $2.992(9)$ \\
\hline & O36 & $3.112(8)$ & & O33B & $3.146(8)$ \\
\hline & O9 & $3.177(10)$ & & O34B & $3.173(7)$ \\
\hline & $\mathrm{O} 27$ & $3.248(8)$ & & & \\
\hline & O6 & $3.261(8)$ & & & \\
\hline & $\mathrm{O} 28$ & $3.280(7)$ & & & \\
\hline K11 & $\mathrm{O} 27$ & $2.788(8)$ & K11B & O4B & $2.855(7)$ \\
\hline & $\mathrm{O} 28$ & $2.844(7)$ & & $\mathrm{O} 28 \mathrm{~B}$ & $2.912(9)$ \\
\hline & $\mathrm{O} 7$ & $2.897(9)$ & & O27B & $2.928(9)$ \\
\hline & $\mathrm{O} 36$ & $2.906(8)$ & & O8B & $3.133(8)$ \\
\hline & $\mathrm{O} 35$ & $2.910(8)$ & & O29B & $3.174(8)$ \\
\hline & O8 & $3.226(8)$ & & O30B & $3.196(8)$ \\
\hline & $\mathrm{O} 31$ & $3.304(8)$ & & O36B & $3.240(9)$ \\
\hline & $\mathrm{O} 4$ & $3.345(8)$ & & O35B & $3.273(9)$ \\
\hline & $\mathrm{O} 32$ & $3.365(7)$ & & O32B & $3.369(8)$ \\
\hline & O26 & $3.374(7)$ & & O31B & $3.396(8)$ \\
\hline
\end{tabular}




\section{References}

Burla, M. C., Caliandro, R., Carrozzini, B., Cascarano, G.L., Cuocci, C., Giacovazzo, C., Mallamo, M., Mazzone, A. \& Polidori G. (2015). J. Appl. Cryst. 48, 306-309.

Sheldrick, G. M. (2015). Acta Cryst. C71, 3-8. 


\section{S6. Rietveld refinements using high resolution synchrotron radiation}

The major steps of Rietveld refinements are resumed in Table S6.1 and can be divided in two groups: the first, from $\# 40$ to $\# 235$, starts with the raw model in space group $P 3 c 1$ obtained from 3D ED and addresses various improvements until the introduction of the model in $P 3$ obtained from single crystal refinement, and the second, from \#b10 to \# 20, is concerned with the correct description of peak shape (anisotropic line broadening ALB). Some representative patterns are shown at the end.

Table S6.1. Summary of Rietveld and Le Bail refinements of kaliophilite using high resolution synchrotron powder diffraction data. Peak shape is TCH pseudoVoigt throughout.

\begin{tabular}{|c|c|c|c|c|c|c|c|c|c|}
\hline \# & prog & sg & Ks & $N P$ & parameters (refined/not refined) & $\chi^{2}$ & $R\left(F^{2}\right)$ & conv & parity for $h-k$ \\
\hline 40 & gsas & $P 3 c 1$ & - & 33 & $a-Z-X-Y 24 b k 3 U$ & 935 & 0.34 & Y & \\
\hline 58 & “ & “ & - & 195 & $a-Z-X-Y 24 b k 3 U-162 x(\mathrm{DLS})$ & 444 & 0.20 & $\mathrm{~N}$ & \\
\hline 642 & “ & “ & 3.1 & 127 & $a-Z-X-\underline{Y}-s t-p t-s f 32 b k 3 U-80 x(\mathrm{DLS})$ & 357 & 0.17 & $\mathrm{~N}$ & \\
\hline 235 & “ & $P 3$ & 2.7 & 44 & $a-Z-X-\underline{Y}-s t-p t-s f 32 b k$ & 292 & 0.131 & Y & \\
\hline b10 & FP & “ & 2.3 & 38 & $a-Z-X-Y-Y_{\text {anis }} 27 b k$ & 136 & 0.090 & $\mathrm{Y}$ & $B: 3 n$ \\
\hline $\mathrm{c} 18$ & “ & “ & 3.0 & $10 \mathrm{LB}$ & $a-Z-X-Y-Y_{\text {anis }}-\int \underline{51 b k}$ & 43.1 & 0.00018 & Y & $B-\int: 3 n$ \\
\hline $\mathrm{f} 21$ & “ & “ & 2.8 & $13 \mathrm{LB}$ & $a-Z-X-Y-Y_{\text {anis }}-\int \underline{45 b k}$ & 31.3 & 0.00034 & $\mathrm{Y}$ & $B: 3 n[+1], \int: 3 n$ \\
\hline e20 & “ & “ & 2.8 & 2 & 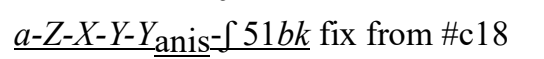 & 117 & 0.089 & Y & $B-\int: 3 n$ \\
\hline
\end{tabular}

NOTES: prog program, sg space group, Ks \% kalsilite impurity, $N P$ number of refined parameters, conv convergence, gsas GSAS (Larson \& Von Dreele, 2004), FP FullProf (Rodríguez-Carvajal, 2001), LB Le Bail refinement (rest is Rietveld), $a$ unit cell(s), $Z$ 20-origin, $X(Y)$ strain(size) component of Lorentzian, st-pt-sf shape anisotropy with subcell (gsas), $Y_{\text {anis }}$ subcell size component (FP), $24 b k 24$ background parameters, $U$ atom displacement parameter, $x$ atom coordinates, DLS restraint geometry for tetrahedra, $B F W H M, \int$ peak shift ( $B$ and $\int$ are the parameters to which the parity condition applies).

\section{Group 1 (structure from $P 3 c 1$ to $P 3$ )}

The starting structure, obtained from 3D ED and preliminarily refined using electron diffraction intensities, had already an ordered $\mathrm{Al} / \mathrm{Si}$ distribution. Once introduced in Rietveld refinement (\#40, $\left.R\left(F^{2}\right)=0.34\right)$, it became immediately clear that there were enormous problems with peak shape, and trials to refine the structure $\left(\# 58, R\left(F^{2}\right)=0.20\right.$ ) showed correlations and divergence even when the tetrahedral distances (T-O and O-O) were restrained. Slight improvements could be achieved introducing shape anisotropy to model ALB and a small fraction of kalsilite as impurity to account for some extra peaks ( $\left.\# 642, R\left(F^{2}\right)=0.17\right)$, but results correspond to false minima recognized by lack of convergence and improbable interatomic distances.

In the meanwhile, SCXRD refinement using data from a twinned crystal allowed to obtain an improved structure in the subgroup $P 3$. These atom parameters, introduced in Rietveld refinement without releasing them, clearly lowered the residual $\left(\# 235, \chi^{2}=292, R\left(F^{2}\right)=0.131\right)$ but the inadequateness of peak shape description became now even more visible and is reflected by the high reduced $\chi^{2}$ value ${ }^{\mathrm{a}}$. The use of a microstrain model (Stephens, 1999) as an alternative to explain ALB was not successful either.

\section{Group 2 (improved modelling of peak shape and final refinement)}

Instead, a substantial improvement was obtained $\left(\# \mathrm{~b} 10, \chi^{2}=136, R\left(F^{2}\right)=0.090\right)$, again without changing atom parameters, if the parity rule $h k l: h-k=3 n(h k l: h-k \neq 3 n)$ for sharp (broad) reflections, found from synchrotron single crystal experiments, was introduced.

The new peak shape model was improved in two (structureless) Le Bail refinements (\#c18, $\chi^{2}=43.1 ; \# \mathrm{f} 21, \chi^{2}$ $=31.3$ ), whose details will be discussed below. The overall parameters obtained in \#c18 were, unchanged, fed into a final Rietveld refinement (\# $\left.20, \chi^{2}=117, R\left(F^{2}\right)=0.089\right)$ where only the scale factors of kaliophilite and

\footnotetext{
${ }^{\text {a }}$ Values for $\chi^{2}$ are high throughout due to the low statistical error of diffracted intensities, a fact well known for work with synchrotron radiation, but $\chi^{2}=292$ is still 10 times higher than the minimum obtained in standard refinement $\left(\chi^{2}=\right.$ 30).
} 
kalsilite were allowed to refine. As expected, the global fit error $\chi^{2}$, but not the structural error $R\left(F^{2}\right)$, dropped with respect to step \#b10.

The structural residuals of the final result $\left(\# \mathrm{e} 20, R\left(F^{2}\right)=0.089, R(F)=0.071\right)$ compare well with their counterparts from single crystal refinement $(\mathrm{w} R 2=0.182, R 1=0.090)$, a confirmation which is independent from any bias introduced by twin laws. In addition, Rietveld refinement gave at least three new informations: (a) more representative (whole phase) and precise (synchrotron radiation) values for the unit cell parameters, (b) the detection of kalsilite as an impurity in Colle Cimino kaliophilite, and (c) a wealth of details on microstructure which is discussed below.

\section{Interpretation of line shape and displacement (microstructure)}

The description of peak shape and position was addressed, without any bias coming from crystal structure, using full pattern Le Bail refinements (\#c18 and \#f21 in Table S6.1) as well as single peak fittings of some selected reflections.

In experiment $\# \mathrm{c} 18\left(\chi^{2}=43.1\right)$, ALB was simulated using the $h-k=3 n$ parity rule that divides reflections into two groups with different Lorentzian contributions to the line FWHM. Inspection of the patterns (e.g. peak 311 in Fig. S6.3) shows that many broad peaks exhibit a super-Lorentzian shape which cannot be simulated in the $\mathrm{TCH}$ approach of FullProf. A trial to overcome this limitation was made in experiment $\# \mathrm{f} 21\left(\chi^{2}=31.3\right)$ where three groups of reflections $(h-k=3 n, h-k=3 n+1$ and $h-k=3 n-1)$ were defined. With $3 n-1$ and $3 n+$ 1 being lattice equivalent pairs, this is a mathematical trick to create super-Lorentzians which allow an improved Le Bail fit, showing that super-Lorentzian peak shape explains most of the gap from $\chi^{2}=43.1$ to the minimum at about $\chi^{2}=30$, roughly expected also from NAC standard refinement.

A direct check of the parity rule $h k l: h-k=3 n(h k l: h-k \neq 3 n)$ for sharp (broad) reflections is shown in Fig. S6.1 which compares calculated and observed total FWHMs. The observed line widths (blue dots) were obtained from well resolved reflections and clearly distribute over two branches, confirming that the parity rule holds for all reflections. The lower branch ranges from $F W H M=0.013^{\circ}$ to $0.034^{\circ}$ and closely follows the calculated mean (red diamonds) at the same angle, whereas the upper one (about $0.028-0.042^{\circ}$ ) is more dispersed and values are considerably lower than their calculated counterparts, due to the super-Lorentzian form which, at constant area, has a broader base and smaller FWHM.

Outliers can in most cases be explained. As an example, in the upper branch, negative deviations from the mean are seen for various reflections with high --l indices, e.g. 213, 423, 515, 875. This indicates needle shaped domains for the $27 \AA$ cell, elongated parallel to $\mathbf{c}$ in perfect agreement with HRTEM where faults parallel to $\mathbf{c}$ are observed ${ }^{\mathrm{b}}$. A resulting scheme for ALB is given in the drawing at right of Fig. S6.2. Note the difference to the scheme at left which was unable to fit data (experiment \#235).

\footnotetext{
b The shape anisotropy could be modelled, but was far less important than the parity rule for peak width and the two models cannot be applied simultaneously.
} 


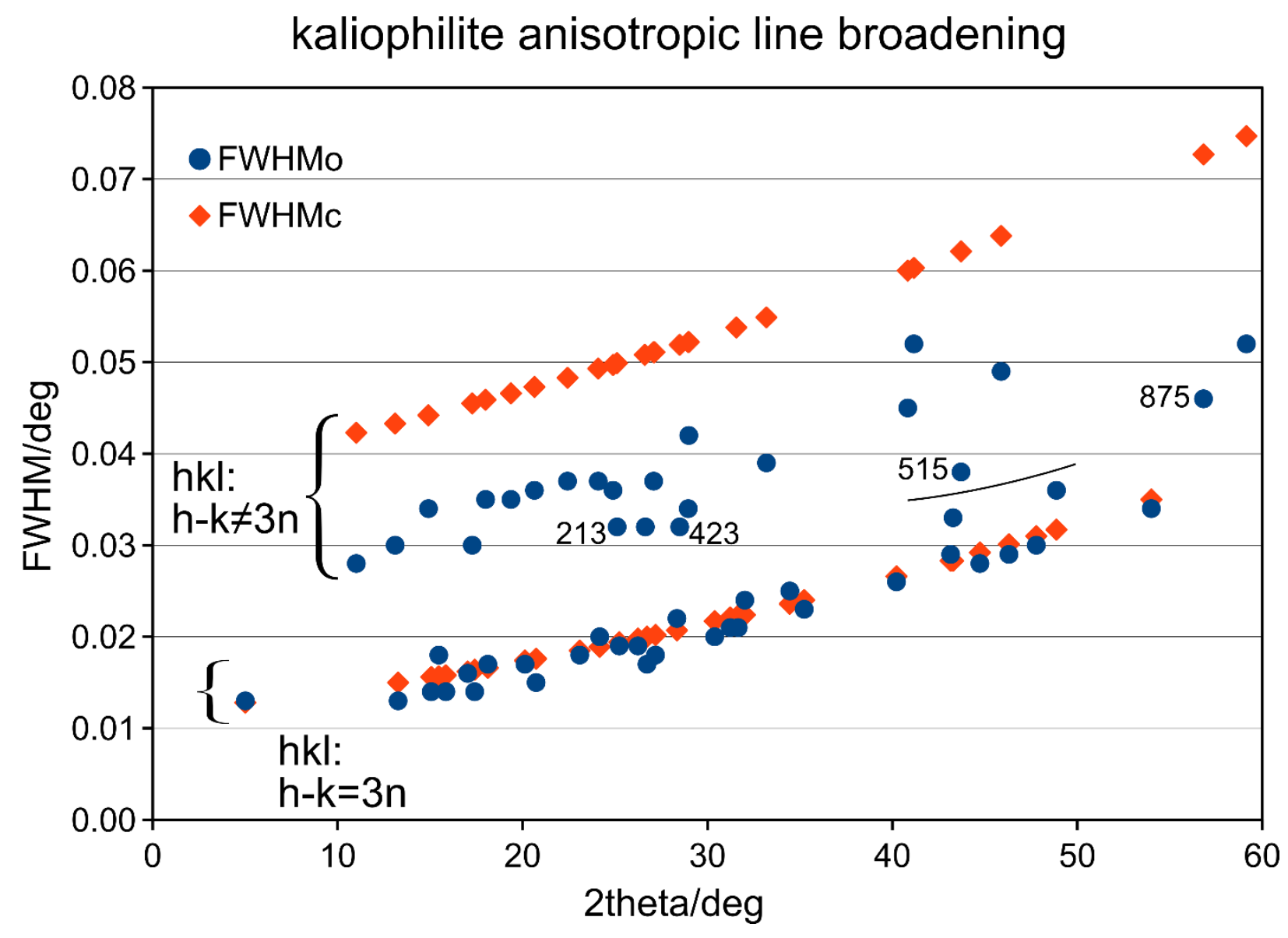

Figure S6.1. Observed (blue) and calculated (\#c18; red diamonds) peak widths for kaliophilite high resolution powder diffraction pattern obtained with synchrotron radiation. For both series, reflections complying with $h k l: h-k=3 n$ are sharper than the remainder. See text for explanations.
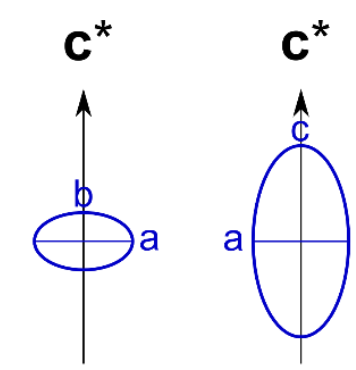

$\mathrm{h}-\mathrm{k}=3 \mathrm{n}$

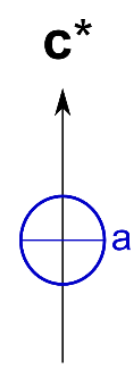

$=3 n$

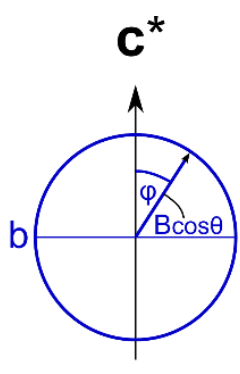

$\neq 3 n$

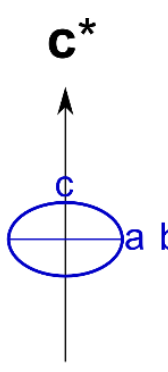

$=3 n$

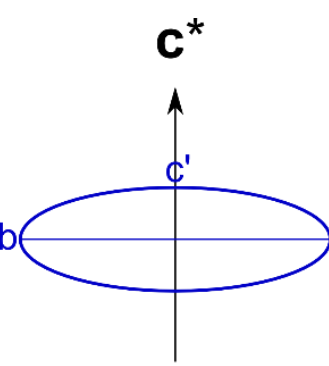

$\neq 3 n$

\section{stacking fault "clay"}

kaliophilite subcell only kaliophilite + shape anisotropy

Figure S6.2. Rotational ellipsoids representing anisotropic line broadening (ALB) for three different hypotheses containing a subcell defined by parity condition $h-k=3 n$. The vector $B \cos \theta=F W H M \cos \theta$ is the $\cos \theta$ corrected line breadth for reflections at inclination $\varphi$ from axis $\mathbf{c}^{*}$. In the first case ("clay"), there are stacking faults (planes orthogonal to $\mathbf{c}^{*}$ ) and the two sets of reflections have the same width orthogonal to $\mathbf{c}^{*}$ but different widths for $\varphi<90^{\circ}$. In the second case (corresponding to the model used in all refinements from \#b10 onwards), widths are independent and different but isotropic within each set. The last case represents the most probable model for kaliophilite, where shape anisotropy (needles parallel to c) is added. Note that, here, the length of the coherent domain in subcell and full cell are likely to be similar (semiaxes $c \simeq c^{\prime}$ ), in contrast to case 1 where the width of reflections perpendicular $\mathbf{c}^{*}$ is common to the two sets. 
The vertical shift $\left(\sim 0.017^{\circ}\right)$ between the two branches is constant, i.e. there is a difference in the size component of line broadening while microstrain remains similar. Domain sizes were estimated using the Scherrer equation with integral breadths $\beta$ obtained from both Le Bail refinement and single peak fits. For the broad reflections, after subtraction of the instrument and Lorentzian strain contributions, one obtains $\beta=0.050^{\circ}$ and $\beta=0.045^{\circ}$, respectively, corresponding to apparent domain sizes of $1360 \AA$ and $1500 \AA$. Such domain sizes apply essentially to $h k l: h-k \neq 3 h$ reflections with low indices (e.g. 211 and 311) and an inclination of $\varphi \simeq 50^{\circ}$ from

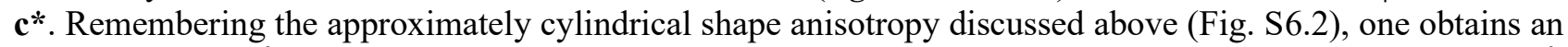
average of $1070 \AA$ for the thickness of the cylinders, in fair agreement with HRTEM results where the $27 \AA$ cell is seen to extend over $200-1200 \AA$ in $\mathbf{a b}$.

Fig. S6.3 shows the intensity patterns of Le Bail and single peak fits for the representative reflections 311 ( $h k l$ : $h-k \neq 3 n)$ and $410(h k l: h-k=3 n)$. Fig. S6.4 reports the best Rietveld fit (\#e20, $\left.\chi^{2}=117\right)$. 

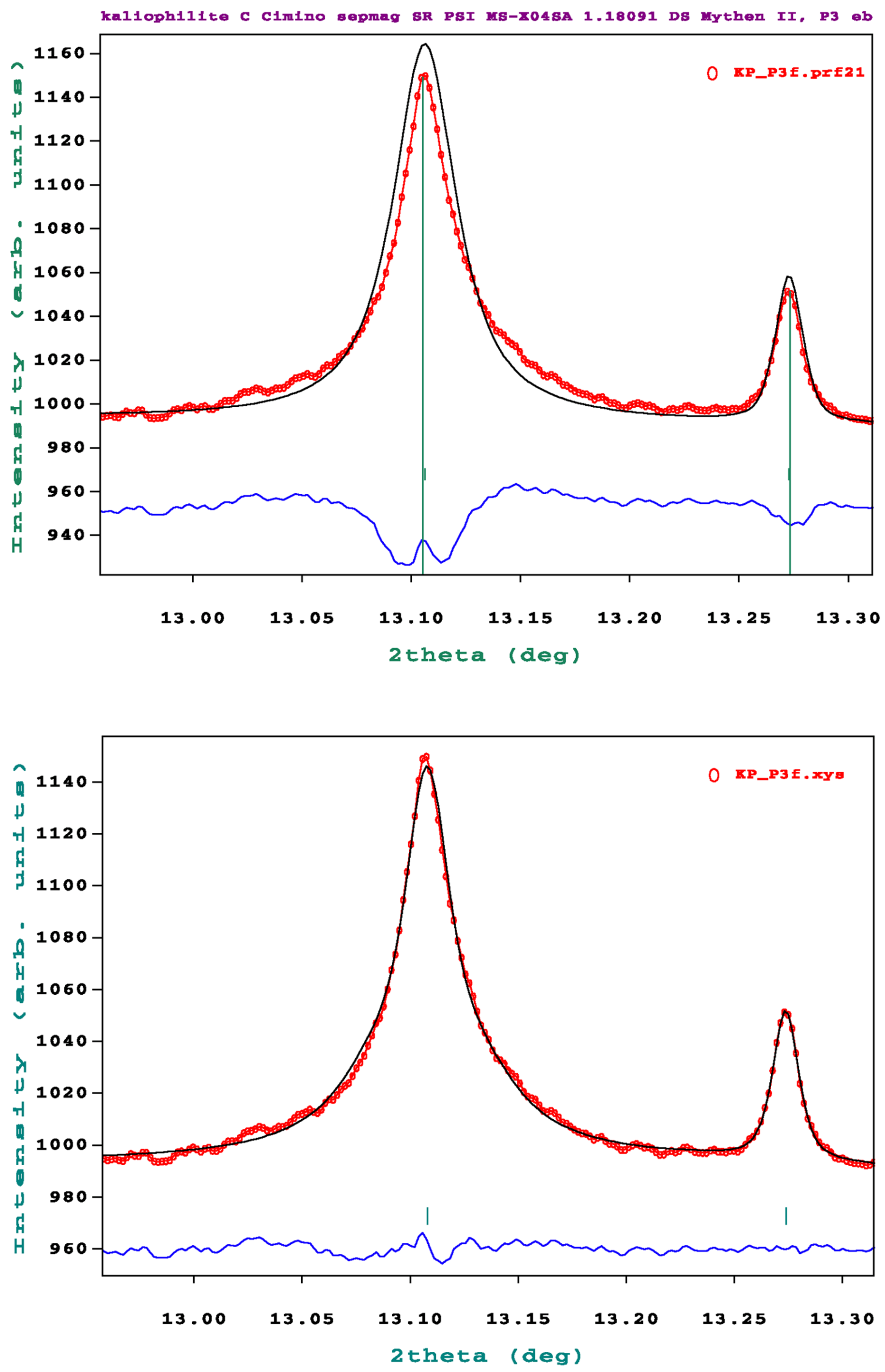

Figure S6.3. Comparison of reflections 311 and 410 from Le Bail (experiment \#f21, top) and single peak fitting (bottom, $\left.\chi^{2}=5.48\right)$. Note the huge improvement with super-Lorentzian shape of $311(\eta=1.29)$. Red dots: observed intensities, black curve: calculated intensities, blue curve: difference; ticks for Bragg positions of kaliophilite are dark green. 


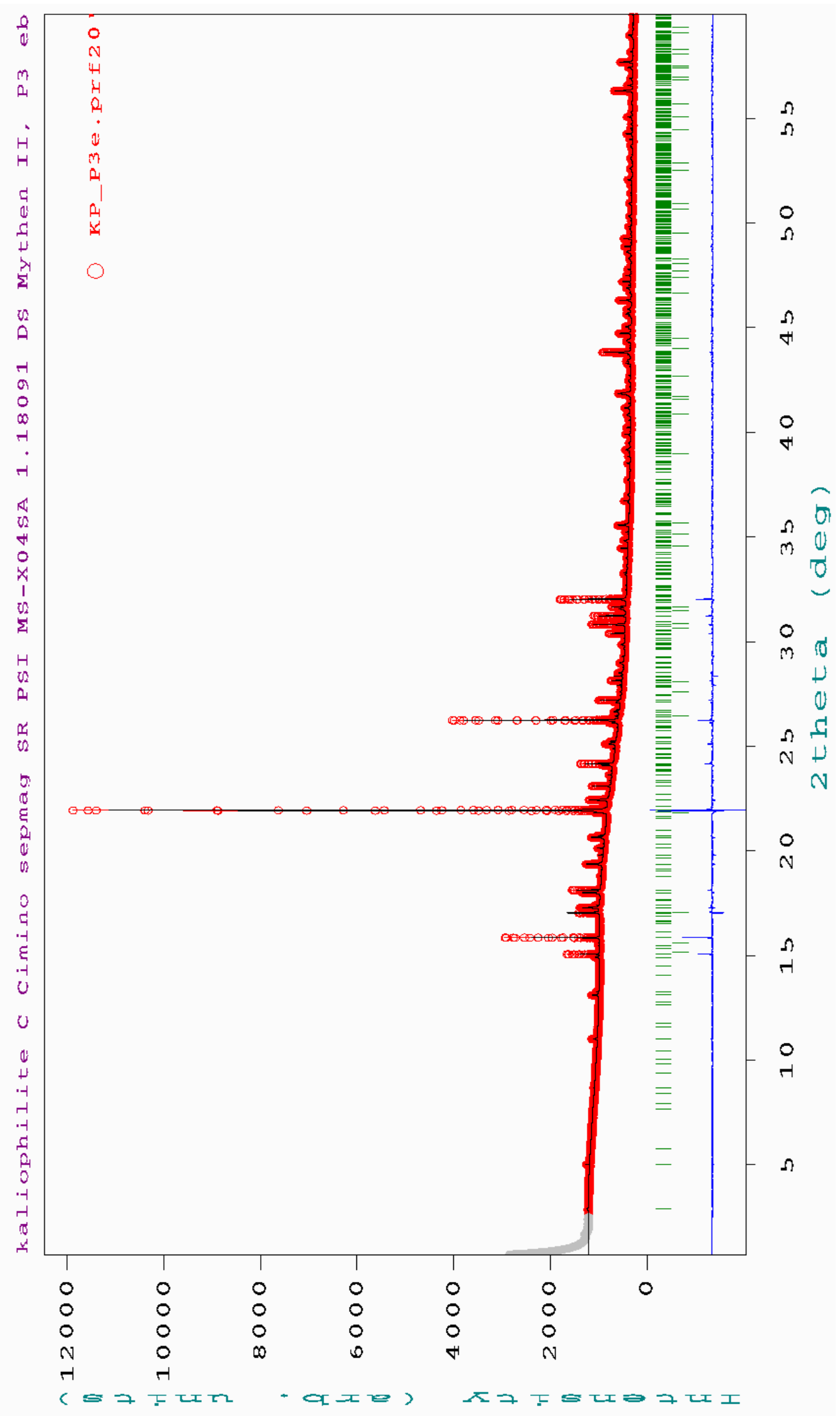

Figure S6.4. Rietveld fit \# 20 for kaliophilite. Red dots: observed intensities, black curve: calculated intensities, blue curve: difference; ticks for Bragg positions are dark green for both kaliophilite and the kalsilite impurity. 
Unit cell parameters of kaliophilite and their errors

For the Colle Cimino sample, the unit cell parameters obtained from Le Bail refinement \#c18 are $a=$ 27.0344(2), $c=8.56362(5) \AA, V=5420.28(9) \AA^{3}$ (experiment $\# \mathrm{f} 21$ gave the same values but slightly lower errors). For the Monte Somma Vesuvius sample, the parameters were obtained from single crystal refinement and are different ( $a=27.0597(16), c=8.5587(6) \AA)$.

Precisions are likely to be overestimated (Herbstein, 2000; David, 2004; Tian \& Billinge, 2011), but there might also be some difference in composition and microstructure. The second phenomenon seems particularly interesting in view of the evident misfit of reflection 110 (Fig. S6.5). A test was therefore designed were reflection 110 was forced to fit observation (note that this reflection has a low intensity and little weight in the global refinement, but it occurs at low angle and is well separated from anything else). Agood fit of the position was obtained for $a=27.051 \AA$ which comes nearer to the Monte Somma value.

In this refinement, peak shape parameters stood almost unchanged at their original values, but the shift parameters changed and all unit cell parameters blew up. In particular, the sine shift parameter changed from 8.6 to $56.2 \mathrm{mdeg}$, which corresponds to sample displacements of about 0.1 and $0.8 \mathrm{~mm}$ along the X-ray beam. The first value comes near to the result for NAC standard refinement (realized at the same date) and is within the expected limits (Gozzo et al., 2010), whereas the second value seems unreasonably high for the experimental setup at the MS-X04SA beamline.

We therefore postulate that the displacement of reflection 110 is due to details of the microstructure which are still to be resolved.

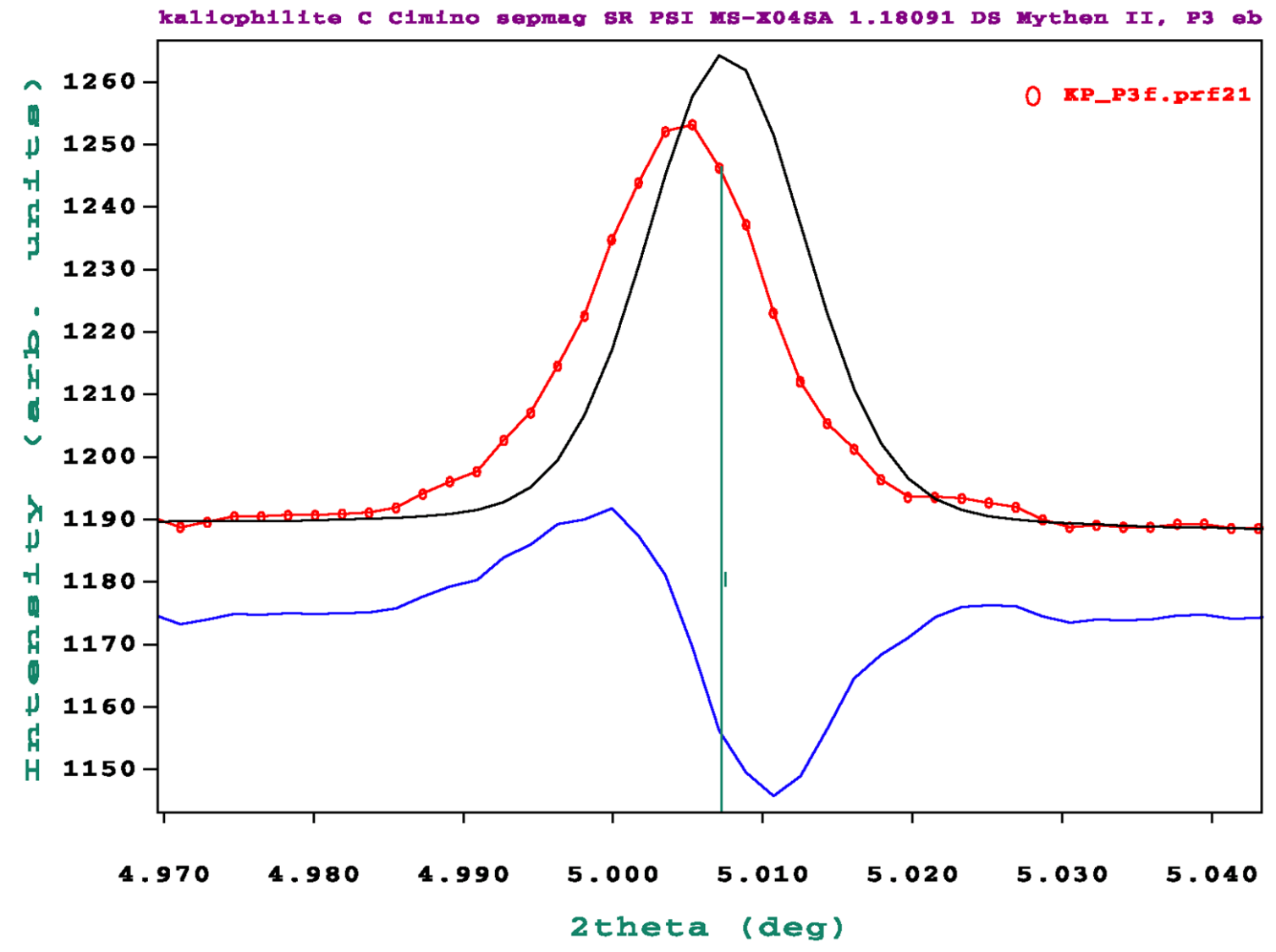

Figure S6.5. Reflection 110, obtained from Le Bail fitting (\#f21). Note the shift of position. Red dots: observed intensities, black curve: calculated intensities, blue curve: difference; ticks for Bragg positions of kaliophilite are dark green. 


\section{References}

David, W. I. F. (2004). J. Appl. Cryst. 37, 621-628.

Gozzo, F., Cervellino, A., Leoni, M., Scardi, P., Bergamaschi, A. \& Schmitt, B. (2010). Z. Kristallogr. 225, 616-624.

Herbstein, F. H. (2000). Acta Cryst. B56, 547-557.

Larson, A. C. \& Von Dreele, R. B. (2004). General Structure Analysis System (GSAS), Los Alamos National Laboratory Report LAUR 86-748.

Rodríguez-Carvajal J. (2001). IUCr Commission on Powder Diffraction Newsletter 26, 12-19.

Stephens, P. W. (1999). J. Appl. Cryst. 32, 281-289.

Tian, P. \& Billinge, S. J. L. (2011). Z. Kristallogr. 226, 898-904. 


\section{S7. Pseudo-extinction rule}

\section{Mathematic derivation of systematic enhancement and weakening of reflections}

The immediately striking features of both single-crystal electron and X-ray patterns are:

1) reflections $h-k=3 n$ are stronger than reflections $h-k \neq 3 n$ for planes with $l$ even;

2) reflections $h-k \neq 3 n$ are stronger that reflections $h-k=3 n$ for planes with $l$ odd;

These features may be intuitively associated with a non-crystallographic translation that becomes evident when describing the kaliophilite structure in terms of corollas containing seven six-membered rings.

The non-crystallographic translation $\mathbf{t}=\left[\frac{1}{3},-\frac{1}{3}, \frac{1}{2}\right]$ relates almost exactly the corollas centred on the ternaries in the unit cell interior (corollas A and B), but it is not valid between any one of these corollas and the corolla centred at the origin (corolla $\mathrm{C}$ ). To prove the effect of this situation on diffraction intensities, we assume that the translation is valid for all atoms, i.e. that for each atom at coordinates $x, y, z$, a translated equal atom will occur at coordinates $x+\frac{1}{3}, y-\frac{1}{3}, z+\frac{1}{2}$.

For $2 N$ atoms in the unit cell, the structure factor can then be written as

$$
\begin{gathered}
F_{h k l}=\sum_{j=1}^{N} f_{j} \exp [2 \pi i(h x+k y+l z)]+\sum_{j=1}^{N} f_{j} \exp \left\{2 \pi i\left[h\left(x+\frac{1}{3}\right)+k\left(y-\frac{1}{3}\right)+l\left(z+\frac{1}{2}\right)\right]\right\}= \\
=\sum f_{j} \exp [2 \pi i(h x+k y+l z)]\left\{1+\exp \left[2 \pi i\left(\frac{1}{3} h-\frac{1}{3} k+\frac{1}{2} l\right)\right]\right\} .
\end{gathered}
$$

The second factor in brackets corresponds to $F_{h k l}=0$ for $\left(\frac{1}{3} h-\frac{1}{3} k+\frac{1}{2} l\right)=\frac{2 n+1}{2}$, and to a maximum of the structure factor for $\left(\frac{1}{3} h-\frac{1}{3} k+\frac{1}{2} l\right)=n$.

Let us separate two possible cases:

1) For $l=2 m$ the former condition (structure factor equal to zero) is never fulfilled for any values of $h$ and $k$. It means that in general the reflections have intensities different from zero. However, the latter condition imposes that the reflections have maximum intensity for $h-k=3 n$.

2) For $l=2 m+1$ the situation is reversed in the sense that $h-k=3 n$ corresponds to a null structure factor. The latter condition is never fulfilled, i.e. for $h-k \neq 3 n$ we never obtain the maximum value, but intermediate ones.

These results explain the maximum intensities for $h k l: h-k=3 n$ and $l$ even, and the almost zero intensities for $h k l: h-k=3 n$ and $l$ odd, as observed in our experimental diffraction patterns.

We can also calculate in detail the relative values of all four groups of intensities when we simplify the problem to a trigonal unit cell containing only two atoms, at the positions $\frac{1}{3},-\frac{1}{3}, 0$ and $-\frac{1}{3}, \frac{1}{3}, \frac{1}{2}$. 
The structure factor can then be written as

$F_{h k l}=f_{j}\left\{\exp \left[i 2 \pi\left(h \frac{1}{3}-k \frac{1}{3}+l * 0\right)\right]+\exp \left[i 2 \pi\left(-h \frac{1}{3}+k \frac{1}{3}+l \frac{1}{2}\right)\right]\right\}$

and

$\frac{F_{h k l}}{f_{j}}=\exp \left[i 2 \pi \frac{1}{3}(h-k)\right]+\exp \left[-i 2 \pi \frac{1}{3}(h-k)\right] \exp (i \pi l)$.

For $l$ even, equation (2) results in:

$\frac{F_{h k l}}{f_{j}}=\exp \left[i 2 \pi \frac{1}{3}(h-k)\right]+\exp \left[-i 2 \pi \frac{1}{3}(h-k)\right]$.

For $l$ odd, equation (2) results in:

$\frac{F_{h k l}}{f_{j}}=\exp \left[i 2 \pi \frac{1}{3}(h-k)\right]-\exp \left[-i 2 \pi \frac{1}{3}(h-k)\right]$.

After substitution for parity rule $k=h-3 n$, equation (3) becomes:

$\frac{F_{h k l}}{f_{j}}=\exp \left[i 2 \pi \frac{1}{3}(3 n)\right]+\exp \left[-i 2 \pi \frac{1}{3}(3 n)\right]=2$

while equation (4) becomes:

$\frac{F_{h k l}}{f_{j}}=\exp \left[i 2 \pi \frac{1}{3}(3 n)\right]-\exp \left[-i 2 \pi \frac{1}{3}(3 n)\right]=0$.

Conversely, for reflections that do not obey the parity rule, i.e. reflections for which $k=h-3 n \pm 1$, equation (3) becomes:

$\frac{F_{h k l}}{f_{j}}=\exp \left[i 2 \pi\left(n \pm \frac{1}{3}\right)\right]+\exp \left[-i 2 \pi\left(n \pm \frac{1}{3}\right)\right]=-1$

while equation (4) becomes:

$\frac{F_{h k l}}{f_{j}}=\exp \left[i 2 \pi\left(n \pm \frac{1}{3}\right)\right]-\exp \left[-i 2 \pi\left(n \pm \frac{1}{3}\right)\right]= \pm i \sqrt{3}$.

Passing from structure factors to intensities, equations (5), (6), (7) and (8) show that, in layers with $l$ even, the normalized intensities take the value 4 for reflections complying with the parity rule $h-k=3 n$, while intensities take value 1 for reflections not complying with such parity rule. Conversely, for layers with $l$ odd, the normalized intensities take the value 0 for reflections complying with the parity rule $h-k=3 n$, while intensities take value 3 for reflections not complying with such parity rule. This is perfectly in agreement with the observed pseudo-extinctions or, more precisely, with the nearly fulfilled non-crystallographic extinctions of kaliophilite. 